\title{
Emergent Goal-Anticipatory Gaze in Infants via Event-Predictive Learning and Inference
}

\author{
Christian Gumbsch \\ University of Tübingen \\ \& MPI for Intelligent Systems \\ Tübingen, Germany \\ christian.gumbsch@uni-tuebingen.de
}

\author{
Maurits Adam \\ University of Potsdam \\ Potsdam, Germany \\ maurits.adam@uni-potsdam.de
}

\author{
Martin V. Butz \\ University of Tübingen \\ Tübingen, Germany \\ martin.butz@uni-tuebingen.de
}

\begin{abstract}
From about 7 months of age onwards, infants start to reliably fixate the goal of an observed action, such as a grasp, before the action is complete. The available research has identified a variety of factors that influence such goal-anticipatory gaze shifts, including the experience with the shown action events and familiarity with the observed agents. However, the underlying cognitive processes are still heavily debated. We propose that our minds (i) tend to structure sensorimotor dynamics into probabilistic, generative event-predictive and event-boundary-predictive models, and, meanwhile, (ii) choose actions with the objective to minimize predicted uncertainty. We implement this proposition by means of event-predictive learning and active inference. The implemented learning mechanism induces an inductive, event-predictive bias, thus developing schematic encodings of experienced events and event boundaries. The implemented active inference principle chooses actions by aiming at minimizing expected future uncertainty. We train our system on multiple object-manipulation events. As a result, the generation of goal-anticipatory gaze shifts emerges while learning about object manipulations: the model starts fixating the inferred goal already at the start of an observed event after having sampled some experience with possible events and when a familiar agent (i.e., a hand) is involved. Meanwhile, the model keeps reactively tracking an unfamiliar agent (i.e, a mechanical claw) that is performing the same movement. We qualitatively compare these modeling results to behavioral data of infants and conclude that event-predictive learning combined with active inference may be critical for eliciting goal-anticipatory gaze behavior in infants.
\end{abstract}

Keywords: infancy, goal-anticipatory gaze, computational model, event cognition, active inference 


\section{Introduction}

Already during the first year of life, infants appear to develop a rudimentary understanding that human actions are directed towards goals. One associated paradigm investigates the development of goal-anticipatory gaze shifts. In eye-tracking studies, infants watch video sequences depicting action events, e.g., a hand reaching for an object. If an infant looks at the goal of the shown event, such as a to-be grasped object, before the movement, such as a reach, is completed, the infant successfully anticipated the goal of the event and thus apparently recognized the goal-directedness of the action. The development of this ability seems to be supported by various factors, such as familiarity with an observed event and the involved agent (Cannon \& Woodward, 2012; Kanakogi \& Itakura, 2011), the motor ability to perform the movement themselves, behavioral cues that indicate agency, and the saliency of the produced effect (Adam, Reitenbach, \& Elsner, 2017; Adam \& Elsner, 2018; Kanakogi \& Itakura, 2011). Despite the rather large conglomerate of findings, the involved internal representations and computational mechanisms are still mostly unknown and have been characterized only descriptively so far (see e.g. Gredebäck and Falck-Ytter (2015) for a review).

In this paper, we propose that goal-anticipatory gaze shifts emerge in infants from two interplaying factors: (i) internally developing probabilistic generative models of action events and transitions between events, and (ii) the overall 'objective' of the brain to minimize uncertainty in its currently activated generative models, that is, in its internal estimates about what is currently happening and what is about to happen in the outside environment. ${ }^{1}$ In support of this proposal, we modeled the emergence of goal-anticipatory gaze shifts in infants, merging recent insights from event-predictive cognition and approximate free-energy minimizing inference. The modeling system first learned about different object interactions in a virtual scenario, such as $<$ reaching for an object $>$ or $<$ transporting an object $>$. For each type of object interaction, it learned distinct event schematic, generative models, which encode the event boundary and the event dynamics in a probabilistic manner. Throughout training, we put our system in experimental conditions, similar to how goal-anticipatory gaze shifts are tested in infants. The system was shown familiar action events (reaching) performed by agents that typically perform this kind of action (hand) or by unfamiliar agents (mechanical claw). We demonstrate that when the system used active inference, that is, it chose its gaze to minimize predicted uncertainty (Friston et al., 2015, 2016), the system showed similar gaze behavior as previously found in infants. Moreover, we analyze how experience with the events affected the gaze behavior, and we qualitatively compare these modeling results to behavioral data of different age groups. Seeing the closely, qualitatively fitting results, we hypothesize that event-predictive, inductive learning biases combined with active inference principles appear to be highly important for enabling the inference of goals while observing others interacting with their environment.

The remainder of this paper is structured as follows. We first give an overview on goal-anticipatory gaze shifts and motivate our generative, event-predictive modeling approach. Next we provide the algorithmic

\footnotetext{
${ }^{1}$ To be more precise, the activated internal estimates usually consider only those aspects of the outside environment that are deemed relevant. Moreover, note that the objective to minimize uncertainty causes the system to attempt to maximize its precision estimates of its internal currently activated event-predictive encodings.
} 
details of our learning and inference model. Section 5 evaluates the model. We summarize and conclude with a discussion of the results and its implications.

\section{Goal-anticipatory gaze behavior in infants}

Looking behavior is one of the first behaviors in human development, and as such, has been widely used to investigate how infants form expectations about observed goal-directed actions (e.g., Fantz, 1958; Gredebäck, Johnson, \& von Hofsten, 2010). A classical measure to capture these expectations is the assessment of infants' looking-times to a still-image of a goal state after an action has been completed (e.g., Woodward, 1998). However, because looking-times are usually measured after the action has been completed, and are measured with low spatial and temporal resolution (Daum, Attig, Gunawan, Prinz, \& Gredebäck, 2012), possible expectations that the infants might have formed can only be inferred post-hoc. The focus of the present paper is on explaining whether and how infants of certain age form goal expectations during the observation of an action that is still unfolding. The production of goal-anticipatory gaze shifts is cognitively demanding (Gredebäck \& Falck-Ytter, 2015) and requires measures with high spatial and temporal resolution, such as eye tracking technology. The research paradigm is mainly based on a seminal eye tracking study in which adult participants tended to shift their gaze to a to-be-attained goal before the goal was actually accomplished in a block-stacking task (Flanagan \& Johansson, 2003). Additionally, such anticipatory gaze behavior occurred when participants performed the task themselves and when they observed someone else performing it. This was taken as evidence for the so-called direct-matching hypothesis: observers visually track ongoing actions based on not only visual features but also on motor-grounded predictive encodings of the perceived actions (e.g., Gallese, Fadiga, Fogassi, \& Rizzolatti, 1996; Rizzolatti, Fadiga, Gallese, \& Fogassi, 1996; Rizzolatti, Fogassi, \& Gallese, 2001). The motor representations are thought to include instructions for the visual system to produce anticipatory gaze shifts, which result in similar gaze behavior during action execution and observation (e.g., Gredebäck \& Falck-Ytter, 2015).

In a study supporting the idea of a direct-matching process during infants' action observation, 6- and 12-month-old infants as well as adults observed how toys moved into a container, either being grasped and transported by a human or floating into the container without observable cause (Falck-Ytter, Gredebäck, $\&$ von Hofsten, 2006). The data revealed anticipatory gaze behavior in the human agent condition for the 12-month-olds and the adults, but not in the self-propelled condition or for the 6-month-olds. Building on these results, several studies replicated and extended these findings. From about 7 months of age onwards, infants show goal-anticipatory gaze shifts when observing simple human grasping actions, while they keep reactively tracking unfamiliar back-of-hand actions or unfamiliar agents such as mechanical claws or self-propelled spoons (Adam et al., 2016; Cannon \& Woodward, 2012; Gredebäck \& Melinder, 2010; Kanakogi \& Itakura, 2011; Krogh-Jespersen \& Woodward, 2014). Moreover, infants' motor abilities and the amount of experience with certain actions are positively correlated with the infants' goal anticipations during observation of the 
respective actions (Cannon, Woodward, Gredebäck, von Hofsten, \& Turek, 2012; Gredebäck \& Melinder, 2010; Kanakogi \& Itakura, 2011).

As an interpretation for the aforementioned results, Elsner and Adam (2021) argue that actions can be perceived as events with a threefold structure. For example, in an unfolding grasping event, an observer will first see the initial phase of the action, including visual features of the agent and the goal object. Then, the grasping event will move into a dynamic phase, where the movement of the agent towards the goal can be perceived. Finally, the grasping event will move into its end phase or end state, where the agent typically arrives at the goal object and manipulates it. This then concludes the grasping event and starts the next observable event.

According to Elsner and Adam (2021), an observer will draw on the available information from these different phases to reason about the goal-directedness of the observed event. During the initial phase and the dynamic phase, only incomplete information about the unfolding event is available, because the goal has not yet been achieved. Therefore, in order for the observer to correctly identify the goal ahead of time, they have to draw on stored top-down event knowledge that is based on prior experience with similar actions or the same action as the one that is currently observed. If, however, no such top-down knowledge is available, the observer can also draw on observable bottom-up information from the action event itself. This entails, for example, the agent's behaviour, because the agent might act in ways or manipulate the goal object in ways that help the observer to reason about the action goal and to also store this new knowledge as top-down information for future observations. This line of reasoning fits well to prior research showing that infants tend to anticipate goals more successfully when they have prior experience with the agent or the action, but are also able to predict the goal of unfamiliar agents or actions when bottom-up information is provided during action observation (e.g. Adam et al., 2017; Adam \& Elsner, 2018; Biro, 2013).

\section{A Developing Event-predictive Inference Model}

Recent theories from different subfields of cognitive science suggest that humans tend to organize their sensorimotor experience by means of hierarchically structured, event encodings (Butz, 2016, 2017; Butz, Achimova, Bilkey, \& Knott, 2021; Zacks, Speer, Swallow, Braver, \& Reynolds, 2007; Zacks \& Tversky, 2001) A crucial characteristic of an event is that it is perceived to have a beginning and an end, i.e., an event boundary (Zacks \& Tversky, 2001). In between two event boundaries, the event unfolds relatively uniformly and, in principle, predictably (Zacks et al., 2007). For instance, a <reaching $>$ event typically starts with the initiation of an arm movement, while it ends when the hand has reached the object, grasping it. Between these boundaries, the hand typically follows a straight trajectory towards the object. Evidence for such event representations stems from different disciplines and can be found on different levels of processing, ranging from sensorimotor activations to semantic and linguistic representations (Baldwin \& Kosie, 2021; Butz et al., 2021; Cooper, 2021; Franklin, Norman, Ranganath, Zacks, \& Gershman, 2020; Kuperberg, 2021). 


\subsection{Generative, event-predictive learning and inference}

From a behavioral perspective, the Theory of Event Coding (TEC) (Hommel, Müsseler, Aschersleben, \& Prinz, 2001; Hommel, 2009, 2015) suggests that actions and their effects are encoded in a common format, i.e., event codes. Event codes develop from action-effect learning, which initially starts with reflex-like, behavioral exploration (Elsner \& Hommel, 2001; Hommel, 2015). At a later stage of behavioral learning they can be used to infer anticipatory behavior: By considering a desired action-effect, the agent activates the linked event code, which automatically also activates the associated motor activity (Elsner \& Hommel, 2001). According to TEC, these action-effects are not restricted to invoking own behavior, but can also be utilized for action understanding, in which case an action observation activates the own event production code.

From an observational perspective, Event Segmentation Theory (EST) (Zacks et al., 2007; Zacks \& Swallow, 2007) is based on the evidence that humans tend to consistently segment perceived streams of information, exhibiting good agreement amongst each other about when event boundaries occur (Zacks \& Tversky, 2001). Event boundaries essentially correspond to significant changes in the unfolding interaction dynamics, which often correspond to subgoals in environmental interactions (Zacks et al., 2007). According to EST, this shared perception of distinct events is the result of internal event models that guide human perceptual processing (Radvansky \& Zacks, 2014; Zacks et al., 2007). Event models are hierarchically organized, generative models that encode entities (such as agents and patients), their sequence of actions, and the resulting consequences in a spatiotemporal framework (Radvansky \& Zacks, 2014; Stawarczyk, Bezdek, \& Zacks, 2021). During the perception of an ongoing event, a subset of event models is active, predicting how the current events are going to unfold, and what will likely be perceived next (Franklin et al., 2020; Zacks et al., 2007). At event boundaries, current event-respective predictions will produce large transient errors, entailing significant changes of the activated event-predictive models.

In sum, humans appear to perceive temporal activity in terms of events. The outlined theories imply particular properties of the underlying event-predictive encoding schemata:

- Event dynamics model: There is general agreement that event encodings are generative, that is, they model how an event typically unfolds, predicting perceptual information dynamics and correlating those with dynamics-influencing motor activities.

- End condition: EST additionally implies that event schemata predict the typical end-effects, which may be equated with final behavioral action effects (e.g., holding an object), which fittingly can serve as desired goals or subgoals.

- Start condition: Finally, event schemata are thought to specify particular preconditions necessary for an event to commence.

It has been shown that such event-predictive encodings can be learned in a dedicated manner, when endowing the learning system with suitable inductive event-predictive biases (Butz, Bilkey, Humaidan, Knott, \& Otte, 

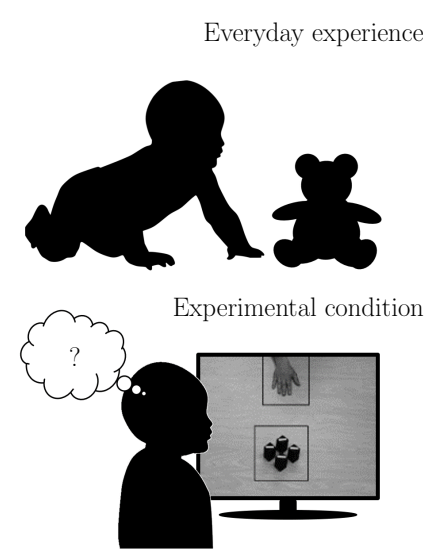

Position predictions

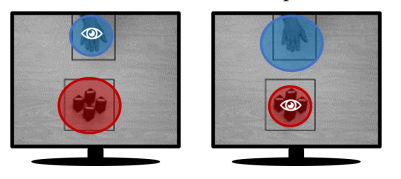

(a) $<6$-month-olds
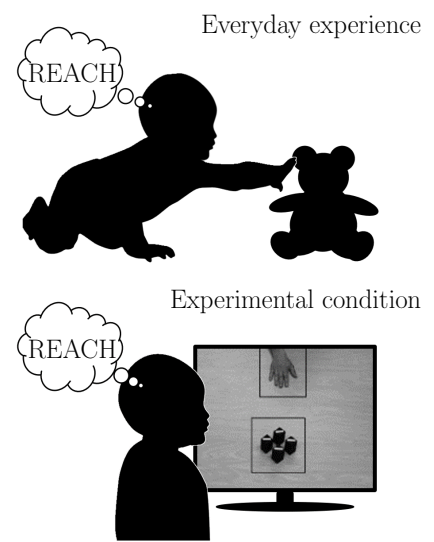

Position predictions

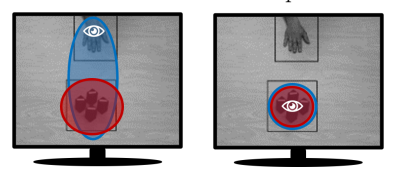

(b) 12-month-olds (hand)
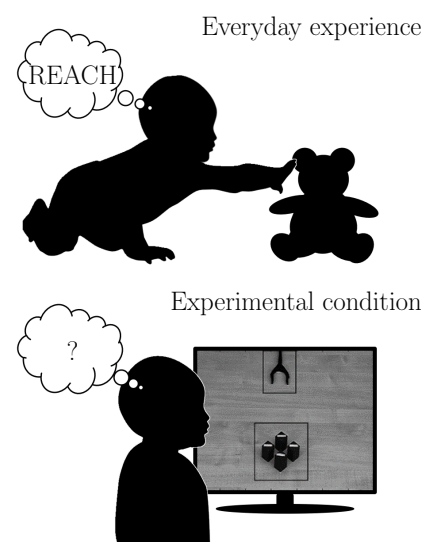

Position predictions
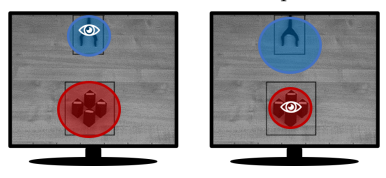

hand/claw position object position

(c) 12-month-olds (claw)

Figure 1: Illustrations of our model's hypotheses about experimental findings on goal-prediction for (a) infants younger than 6 months, (b) for 12-month-old infants watching reaching motions done by hands, and (c) for 12-month-old infants watching reaching motions by mechanical claws. White eye symbols visualize gaze, colored circles visualize position predictions and their confidence (blue for the reaching hand, red for the target position). The screenshots are taken from Adam et al. (2016).

2019; Franklin et al., 2020; Gumbsch, Butz, \& Martius, 2019). Here, we train separate event-predictive models for individual interaction events as well as for transitions from one event to another.

Our generative event-predictive modeling perspective can be seamlessly merged with the Free Energy and active inference principles of cognition (Butz, 2016; Friston, 2010; Friston et al., 2015, 2016). It can also be closely linked to both Bayesian filtering (Knill \& Pouget, 2004) and planning as inference (Botvinick \& Toussaint, 2012). Accordingly, event-predictive encodings are inherently probabilistic and generative. While processing sensorimotor information, free energy minimization causes event-predictive activities to adapt, minimizing the deviance between predictions and encountered sensorimotor dynamics. In effect, bottom-up and top-down information are probabilistically integrated, dynamically maintaining, activating, and de-activating currently applicable event encodings. Meanwhile, active inference generates behavior that attempts to minimize the uncertainty within and across current and subsequent events.

\subsection{Inferring goal-predictive gaze via developing event encodings}

We propose a generative, event-predictive learning and inference model which may explain a variety of experimental findings on the development of goal-anticipatory gaze behavior in infants, illustrated in Figure 1. Anticipatory gaze behavior is modeled for a study scenario in which infants of different ages repeatedly observe a simple goal-directed reach-and-grasp event. Our model assumes that infants younger than 6 months 
do not have a well-enough learned event encoding for $<$ reaching $>$. As a result, they keep their gaze on the moving agent, thereby tracking the hand's movement to gain more information about the agent's future position. From about 7 months onwards, when infants start to show goal-anticipatory gaze, we hypothesize that they have developed a sufficiently well-predicting generative event model. Visual cues, such as the appearance of the hand as well as movement-based information, invoke the activation of the event schema for $<$ reaching $>$. The associated encoding of the end condition will predict that the hand will end up at the position of a target object. The 12-month-old infant will thus anticipatorily look at the target object in order to reduce uncertainty about when, where, and how exactly the observed $<$ reaching $>$ event will end. On the other hand, when 12-month-old infants observe a reaching movement by an unknown agent, e.g. a mechanical claw, that does not exhibit any agency-related cues, the infants tend to reactively track the claw (Adam et al., 2017). Our model assumes that, even though these 12-month-olds have learned an event-generative model for $<$ reaching $>$, this event schema will not be activated because some of the associated start conditions are not met.

In this paper, we investigate the validity of our theoretical considerations by implementing and testing the proposed computational model. Our implemented system, which we term Cognitive Action PRediction model

in Infants (CAPRI), learns schematic, generative event encodings for different interaction events such as $<$ reaching for an object $>,<$ transporting an object $>$, etc. Each event is encoded by a simple generative model that predicts likelihood distributions over expected future observations. Meanwhile, the system continuously attempts to decrease uncertainty about the environment. It essentially attempts to infer which event is unfolding and which future events and event boundaries are likely to occur next. As a result, once particular events have been learned sufficiently well, the system, driven by its aim to reduce uncertainty, begins to direct its gaze in an anticipatory, information-gain-oriented manner much like the goal-anticipatory gaze shifts generated by infants at different ages.

\section{Cognitive Action PRediction model in Infants (CAPRI)}

To evaluate our computational assumptions by means of a concrete free energy-based inference formalization and an actual first implementation of the formalism, we assume that we have a system that 'lives' in a virtual world. The system interacts with its world in every time step $t$ by, first, receiving an observation $o(t)$ and, second, performing an action according to a policy $\pi(t)$, which activates particular motor behavior, such as fixating a particular location in space or moving the hand towards an object. In our scenario, where the agent is an observer, policies correspond solely to gaze behavior.

\subsection{Learning of event schemata}

CAPRI learns event schemata that consist of three encodings: a start condition, the event dynamics model, and an end condition. All components are encoded as probabilistic models, that is, in the form of 


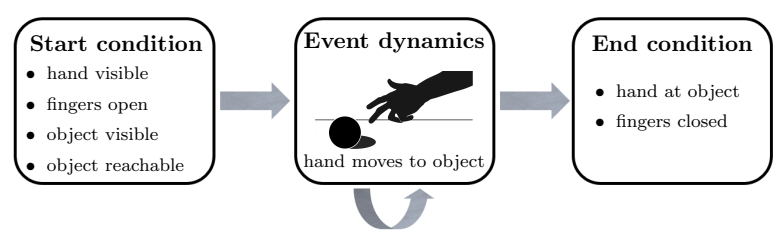

(a)

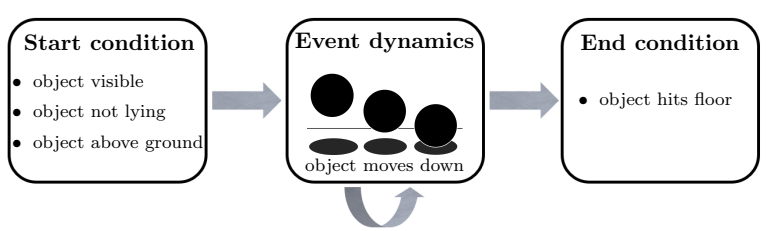

(b)

Figure 2: Illustrations of two event schemata. An event schema is composed of three components: A start condition, an event dynamics model, and an end condition. The graphs exemplarily specifies potential encodings of two events: Reaching (a) and Falling (b).

likelihood distributions.

The start condition $P_{e_{i}}^{\text {start }}$ models the likelihood $P_{e_{i}}^{\text {start }}(o(t) \mid \pi(t-1))$, with the observation $o$, the policy $\pi$, and time $t$. For example, a <reaching $>$ event might typically start with an observation $o(t)$ that contains a hand that just started to move towards a reachable object (cf. Figure 2a). This encoded probability density of the corresponding observation will depend on the gaze location $\pi(t-1)$.

The event dynamics model $e_{i}$ encodes the likelihood of an observation $o(t)$ given the last observation $o(t-1)$ and previously used policy $\pi(t-1)$, that is, eye gaze location: $P_{e_{i}}^{\text {event }}(o(t) \mid o(t-1), \pi(t-1))$. For example, during a $<$ reaching $>$ event the position of the hand at a certain time step $t$, described by observation $o(t)$, is expected to be closer to the object than during the preceding time step $t-1$, described by observation $o(t-1)$ (Figure 2a).

The end condition $P_{e_{i}}^{e n d}$ encodes the likelihood $P_{e_{i}}^{e n d}(o(t) \mid o(t-\kappa), \pi(t-1))$, with the observation $o$, policy $\pi$, time $t$, and a retrospective time horizon $\kappa \geq 1$. Thus, the end condition $P_{e_{i}}^{\text {end }}$ models the likelihood that an observation $o(t)$ occurs at the end of event $e_{i}$ given the last policy $\pi(t-1)$ and some previous observation $o(t-\kappa)$, which lies $\kappa$ time steps in the past. For our reaching example (Figure 2a), this means that at the end of a $<$ reaching $>$ movement at time $t$ the hand position, captured by the observation $o(t)$, is at the same location as the reachable object, which can be predicted by some previous observation $o(t-\kappa)$.

Thus, for every event $e_{i}$ CAPRI learns three separate likelihood distributions over sensory space. All distributions are modeled as multivariate Gaussians, which the system learns to encode by means of Mixture Density Networks (MDNs) (Bishop, 2006) (details in Supplementary Material Section 8.2). During training, which is composed of multiple episodes $E$, the system learns MDNs in a supervised manner. Each episode $E$ can consist of one or multiple events, i.e., $E=\left(e_{i}, e_{j}, \ldots, e_{z}\right)$. When an event $e_{i}$ starts at time $t_{0}$, the starting condition $P_{e_{i}}^{\text {start }}$ is updated using $\pi\left(t_{0}-1\right)$ as an input and $o\left(t_{0}\right)$ as the nominal output. At every time step $t$ during event $e_{i}$, the event dynamics model $P_{e_{i}}^{e v e n t}$ is updated using $o(t-1)$ and $\pi(t-1)$ as an input and $o(t)$ as the nominal output. When an event $e_{i}$ ends at time step $t_{\tau}$, the end condition $P_{e_{i}}^{\text {end }}$ is updated $\tau-1$ times. $P_{e_{i}}^{e n d}$ is updated using $o\left(t_{\tau}\right)$ as nominal output and $\pi\left(t_{\tau}-1\right)$ and $o\left(t_{\kappa}\right)$ as inputs with $t_{\kappa} \in\left[t_{0}, \ldots, t_{\tau}-1\right]$. These multiple model updates do not only increase the training data for the end condition, but also allow CAPRI to learn to generally encode how an event ends, given any observation that had previously occurred during this event. 
After successful training, CAPRI is able to predict the likelihood of an observation occurring at the beginning, end, or during an event $e_{i}$. Let's assume the system has two event models $e_{\text {fall }}$ and $e_{\text {reach }}$, encoding $<$ An object is falling $>$ and $<\mathrm{A}$ hand is reaching for an object $>$, respectively (see Figure 2). When the system perceives $<$ a ball that just rolled over the edge of a table $>$, the start condition $P_{e_{f a l l}}^{\text {start }}$ would predict a high likelihood for this particular observation. On the other hand, when the system observes $<$ the ball hitting the floor $>$, the event end condition $P_{e_{\text {fall }}}^{e n d}$ would give a high likelihood for this observation. Thus, the likelihood estimates can be used to infer the probability of an event in the absence of explicit labels about the ongoing events.

\subsection{Event-predictive inference}

During training CAPRI receives supervised information about which event is currently unfolding. In contrast, during testing CAPRI infers at each point in time $i$ probabilities about which event $e_{i}$ currently unfolds given the available sensorimotor information, that is, the sequence of all previous observations $O(t)=(o(t), o(t-1), \ldots, o(0))$ and all performed policies $\Pi(t)=(\pi(t), \pi(t-1), \ldots, \pi(0))$. That is, the system infers $P\left(e_{i}(t) \mid O(t), \Pi(t)\right)$ for every possible event $e_{i}$. This event inference is performed iteratively every time step after executing an action based on policy $\pi(t-1)$ and receiving a new observation $o(t)$ via:

$$
P\left(e_{i}(t) \mid O(t), \Pi(t)\right)=\sum_{e_{j}} P\left(e_{i}(t) \mid o(t), o(t-1), \pi(t-1), e_{j}(t-1)\right) \cdot P\left(e_{j}(t-1) \mid O(t-1), \Pi(t-1)\right) .
$$

Hence, at every time step $t$, the system computes $P\left(e_{i}(t) \mid o(t), o(t-1), \pi(t-1), e_{j}(t-1)\right)$ for every combination of $e_{i}$ and $e_{j}$ to update the event probabilities. We compute $P\left(e_{i}(t) \mid o(t), o(t-1), \pi(t-1), e_{j}(t-1)\right)$ as:

$P\left(e_{i}(t) \mid o(t), o(t-1), \pi(t-1), e_{j}(t-1)\right)=\frac{P\left(o(t) \mid o(t-1), \pi(t-1), e_{i}(t), e_{j}(t-1)\right) \cdot P\left(e_{i}(t) \mid e_{j}(t-1)\right)}{\sum_{e_{h}} P\left(o(t) \mid o(t-1), \pi(t-1), e_{h}(t), e_{j}(t-1)\right) \cdot P\left(e_{h}(t) \mid e_{j}(t-1)\right)}$.

The full derivation and the underlying assumptions can be found in Section 8.8. We set the event transition prior $P\left(e_{i}(t) \mid e_{j}(t-1)\right)=0.9$ for $e_{i}=e_{j}$ and $P\left(e_{i}(t) \mid e_{j}(t-1)\right)=\frac{0.1}{n}$ for $e_{i} \neq e_{j}$ where $n$ is the number of available events. This corresponds to the assumption that once the system is in one event, it typically stays in the same event with a probability of $90 \%$.

To compute the Bayesian posterior $P\left(o(t) \mid o(t-1), \pi(t-1), e_{i}(t), e_{j}(t-1)\right)$, we use the event schemata distributions, which were outlined in Section 4.1. There are two cases for computing this likelihood depending on $e_{i}(t)$ and $e_{j}(t-1)$ : Either the world remains in the same event or an event transition has happened from time $t-1$ to time $t$. When remaining in the same event, $e_{i}=e_{j}$ and the likelihood of observation $o(t)$ can be computed by:

$$
P\left(o(t) \mid o(t-1), \pi(t-1), e_{i}(t), e_{i}(t-1)\right)=P_{e_{i}}^{\text {event }}(o(t) \mid o(t-1), \pi(t-1))
$$


with $P_{e_{i}}^{\text {event }}$ encoding the event-respective dynamics distribution (see Section 4.1).

On the other hand, when $e_{i} \neq e_{j}$, the likelihood needs to be computed by:

$$
P\left(o(t) \mid o(t-1), \pi(t-1), e_{i}(t), e_{j}(t-1)\right)=P_{e_{i}}^{s t a r t}(o(t) \mid \pi(t-1)) \cdot P_{e_{j}}^{e n d}(o(t) \mid o(t-1), \pi(t-1)),
$$

using the start and end conditions $P^{\text {start }}$ and $P^{\text {end }}$ (see Section 4.1). This corresponds to the idea, that the likelihood of the observation $o(t)$ during an event transition from $e_{j}$ to $e_{i}$ is composed of the likelihood of the end condition of $e_{j}$ and the start condition of $e_{i}$ being satisfied.

The process of event inference is initialized at time $t=0$ with

$$
P\left(e_{i}(0) \mid O(0), \Pi(0)\right)=\frac{P_{e_{i}}^{\text {start }}(o(0) \mid \pi(0))}{\sum_{e_{h}} P_{e_{h}}^{\text {start }}(o(0) \mid \pi(0))},
$$

with $P_{e_{i}}^{\text {start }}$ referring to the start condition of event $e_{i}$ (see Section 4.1).

\subsection{Active inference}

Event inference essentially infers probabilistically which event is currently unfolding using the available, previously learned event schemata and the available sensory information. However, sensory information is not some external, unchangeable quantity simply handed to the system. Rather, CAPRI can actively interact with the world by choosing policies that generate actions, which, in turn, affect the next sensory observation. We follow Friston et al. (2015) to compute expected free energy for every policy $\pi$, as: ${ }^{2}$

$$
\begin{aligned}
\widehat{F E}(\pi, t)= & \underbrace{D_{\tau, e_{i}}\left[P\left(o(\tau) \mid e_{i}(\tau), \pi\right) P\left(e_{i}(\tau) \mid O(\tau), \Pi(\tau)\right) \| P(o(\tau) \mid m(\tau))\right]}_{\text {predicted divergence from desired states }} \\
& +\underbrace{E_{\tau, e_{i}}\left[H\left[P\left(o(\tau) \mid e_{i}(\tau), \pi\right)\right] P\left(e_{i}(\tau) \mid O(\tau), \Pi(\tau)\right)\right]}_{\text {predicted uncertainty }},
\end{aligned}
$$

with $D$ the Kullback-Leibler (KL) divergence, serving as a distance measure between two probability distributions, $m$ a generative model of internal state preferences, $E$ the expectation, and $H$ entropy. The expected free energy is computed for a time horizon $\tau$ expanding from the present into the future. Because CAPRI perceives the unfolding sensory information in terms of discrete events, $\tau$ encodes the number of future event boundaries that are considered for estimating future free energy.

Predicted KL-divergence $D$ from desired states is computed based on a motivational generative model $m$, which encodes internal state preferences. $P(o(t) \mid m(\tau))$ specifies an according distribution over observations for particular, current state preferences $m(\tau)$, which are often equated with states of internal homeostasis. This distribution is compared with the expected observations given the system's estimation of events. As a result,

\footnotetext{
${ }^{2}$ Since Friston et al. use a different notation and do not consider internal event estimations $e_{i}$ we provide a derivation of Equation 6 in Section 8.3.
} 


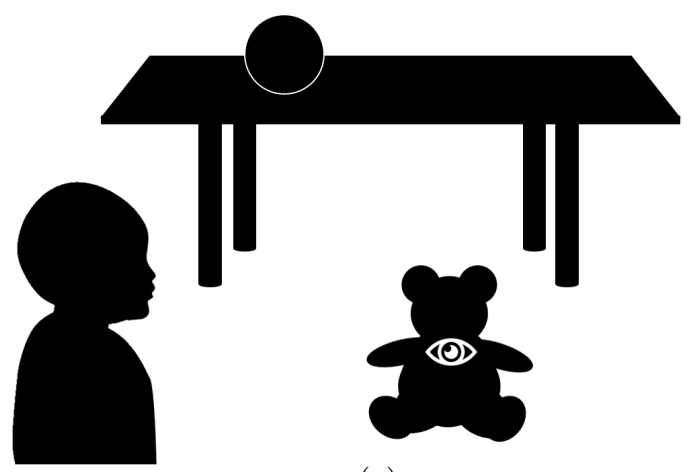

(a)

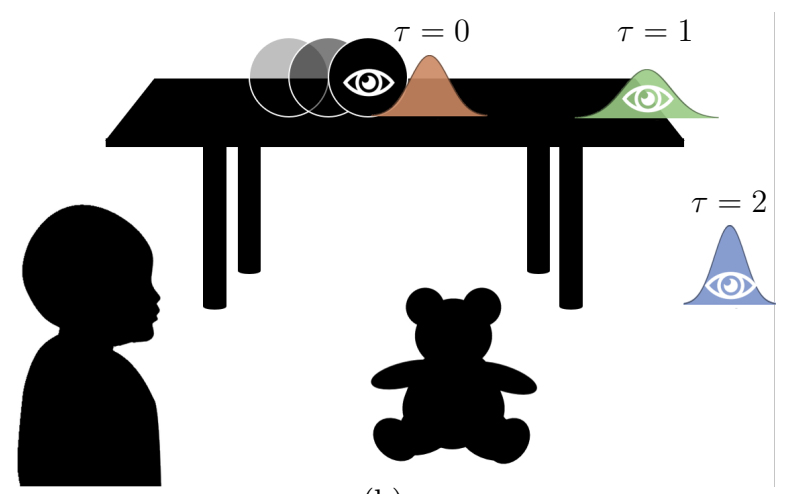

(b)

Figure 3: Illustration of active inference to minimize expected free energy for different policies. In this scene the system can only shift its gaze. Thus, policies $\pi$ encode different gaze positions, which are illustrated by the white eye symbol. (a) Minimizing predicted divergence from desired states, when $m$ desires visual images of teddy bears. (b) Minimizing predicted uncertainty for $\tau=\{0,1,2\}$ when a ball unexpectedly starts to roll. Gaussian bell curves illustrate $P\left(o(t) \mid e_{i}(t), \pi\right)$ for $o$ describing ball positions.

minimizing this term infers behavioral policies that are goal-directed, attempting to generate observations that are in agreement with the desired ones over the specific temporal horizon $\tau$. Let us, for example, assume that we have a system that can only interact with the world by looking at different positions. Hence its policy $\pi$ encodes different gaze positions. Let us further assume that the system currently would like to look at teddy bears. This desire is encoded in $m$. Hence, the distribution of desired observations $P(o(\tau) \mid m(\tau))$ describes visual images of teddy bears. To minimize the divergence between expected and desired observations, the system will choose a policy $\pi$ via which it believes to see teddy bears, as shown in Figure 3a.

The second term of Equation 6, partially competing with the first term, describes predicted uncertainty about future observations in the form of expected entropy, given that policy $\pi(\tau)$ is followed. Choosing a policy that minimizes the second term results in behavior that attempts to maximize certainty about future observations. Consider the scenario shown in Figure 3b. Assuming again that we have a system whose policies correspond to different gaze position, let us assume that our system currently looks at its beloved teddy bear. Meanwhile, it assumes that all other objects and entities in its environment are in the <staying still and doing nothing $>$ event, which we denote by $e_{\text {still }}$. Suddenly a ball on a table in the periphery seems to move. This is highly unexpected since the system assumed that the ball was in event $e_{\text {still }}$. Since the system does not know in which state $e(t)$ the ball is in, the expected entropy over all possible events $e_{i}$ of $E_{\tau, e_{i}} P\left(o(t) \mid e_{i}(t), \pi\right) P\left(e_{i}(t) \mid O(t), \Pi(t)\right)$ is high. By looking at the ball, the system can perceive the most information about the current ball event. Hence, the system would look at the ball because it expects maximum information gain, that is, maximum entropy decrease.

However, it is not enough for the system to know the current event of the ball, which may be denoted by $e_{\text {rolling }}$. Equation 6 implies that the system will infer actions from which it expects to minimize uncertainty about the ball behavior in the near future, considering a temporal horizon $\tau$. The system can use $P_{e_{\text {rolling }}}^{e n d}$ to infer that this event will likely end at the edge of the table. Thus, the system will tend to look at the 
edge of the table next to minimize uncertainty about where and when the ball will go from $<$ rolling $>$ to $<$ falling $>$. Given an even deeper temporal horizon and sufficiently accurate event model beliefs, the system may next look at the floor besides the table to know where and when the ball will go from $<$ falling $>$ to another event, such as <bouncing $>$. Hence, CAPRI's objective to minimize expected future uncertainty can cause anticipatory gaze shifts.

Since we are interested in gaze and assume that attractiveness of the visual stimuli is well-controlled and thus approximately uniform in the modeled experiments, CAPRI focuses only on optimizing its policy, i.e., its gaze locations, for minimizing predicted uncertainty:

$$
\widehat{F E}(\pi, t)=E_{\tau, e_{i}}\left[H\left[P\left(o(t) \mid e_{i}(t), \pi\right)\right] P\left(e_{i}(t) \mid O(t), \Pi(t)\right)\right] .
$$

CAPRI thus chooses the policy $\pi$ for which it expects to minimize this free energy term, based on the active inference principle as follows:

$$
\pi(t)=\arg \min _{\pi_{i}} \widehat{F E}\left(\pi_{i}, t\right) .
$$

For $\tau=0$ this means that CAPRI does not consider future events and it only 'wants' to minimize the entropy of the currently active event dynamics models:

$$
\widehat{F E}(\pi, t)=\sum_{e_{j}} H\left[P_{e_{j}}^{\text {event }}(o(t+1) \mid o(t), \pi)\right] P\left(e_{j}(t) \mid O(t), \Pi(t)\right)
$$

For $\tau=1$, CAPRI not only focuses on the present but also considers next possible event transitions. This means that the system additionally attempts to reduce the uncertainty about the next event boundary, encoded by the event end- and start conditions:

$$
\begin{aligned}
\widehat{F E}(\pi, t)= & \sum_{e_{j}} H\left[P_{e_{j}}^{\text {event }}(o(t+1) \mid o(t), \pi)\right] P\left(e_{j}(t) \mid O(t), \Pi(t)\right) \\
& +\sum_{e_{j}} \sum_{e_{h}} H\left[P_{e_{h}}^{\text {start }}\left(o\left(t^{\prime}\right) \mid \pi\right) P_{e_{j}}^{\text {end }}\left(o\left(t^{\prime}\right) \mid o(t), \pi\right)\right] \cdot P\left(e_{j}(t) \mid O(t), \Pi(t)\right) .
\end{aligned}
$$

When minimizing this quantity, CAPRI attempts to minimize predicted entropy over the currently estimated event dynamics (as in Equation 9) and over all possible next event boundaries. For $\tau=2$ another term can be added that additionally considers one more event boundary into the future, etc.

\section{CAPRI Model Evaluation}

We evaluate our CAPRI implementation in a simple three-dimensional agent-patient-interaction simulation, mimicking infants' probable action experiences. In the following, we first detail the simulation environment and the supervised learning procedure. We then detail the behavior of the system and show that goal-anticipatory gaze behavior indeed selectively emerges over the course of learning. 


\subsection{Simulation setup and learning procedure}

Our simulation always contains two entities - an agent, that is, the hand or another object such as a claw, and a patient, that is, a graspable object. The agent corresponds to the subject of an observed interaction while the patient corresponds to the object of the interaction. At every time step $t$, our system received an 18-dimensional real-valued observation vector $o(t)$, which signals the three-dimensional position of the agent $x^{a}$ and the patient $x^{p}$, their respective three-dimensional velocities $v^{a}$ and $v^{p}$, the relative position of the agent relative to the patient $x^{p-a}=x^{p}-x^{a}$, and the Euclidean distance $d^{a, p}$ between agent and patient. Furthermore, the observation contained a one-dimensional 'representation' of the agent's and the patient's shapes ( $s^{a}$ and $s^{p}$, respectively). This shape signal serves as a simple cue to distinguish hands from other types of entities, such as claws, and, thus, can bias the system's inferred event probabilities. The shape

values for hands, $s_{\text {hand }} \in[0,0.5)$, and claws, $s_{\text {claw }} \in(0.5,1.0]$, representing hands and mechanical claws were randomly determined for every simulation. The shape values for all other entities were randomly sampled from $[0,1]$. Note that the sensory information was position-encoded (starting with the agent followed by the patient and the relative encodings), thus sidestepping the challenge to assign agent and patient roles.

Our system acted choosing different policies, i.e., gaze positions. To do so, the system could choose to focus on one of three significant positions, influencing the standard deviation $\sigma$ of the normally distributed noise levels on agent and patient-related sensory information. The system could look at the agent $\left(\pi_{a}\right)$, yielding $\sigma=0.1$ for all patient-related components $\left(x^{p}, v^{p}, s^{p}, x^{p-a}, d^{a, p}\right)$, but $\sigma=0.01$, for purely agent-based information $\left(x^{a}, v^{a}, s^{a}\right)$. When looking at the patient $\left(\pi_{p}\right)$, the sensory noise scheme of all components was reversed, with $\sigma=0.1$ for the agent and $\sigma=0.01$ for the patient. When looking at neither of the entities $\left(\pi_{n}\right)$, normally distributed noise with $\sigma=0.1$ was added to all sensory components. Thus, we simulated a kind of object-oriented attention, yielding clearer and noisier sensory information about the focused and unfocused entities, respectively, ignoring actual physical distance.

Every event-sequence simulation activated a new pair of agent $a$ and patient $p$ and positioned them randomly in the scenario. Four possible events were considered, mimicking events that may be encountered and produced by infants:

- During a standing still event $\left(e_{\text {still }}\right)$, agent and patient remained motionless for a fixed number of time steps (100 in our simulations), mimicking looking at stationary objects.

- During a randomly directed motion event ( $\left.e_{\text {random }}\right)$, the agent moved constantly in one fixed, but randomly generated direction with decreasing velocity. The event ended when the agent was approximately motionless $\left(v^{a}<0.0005\right)$. This event mimics the observation of rolling or sliding objects, like a toy car.

- During a reaching motion event $\left(e_{\text {reach }}\right)$, a hand agent moved towards the patient with a randomly set, constant velocity. The event ended when the agent approximately reached the patient.

- Hand agents could also perform a transportation event $\left(e_{\text {transport }}\right)$, where agent and patient moved 


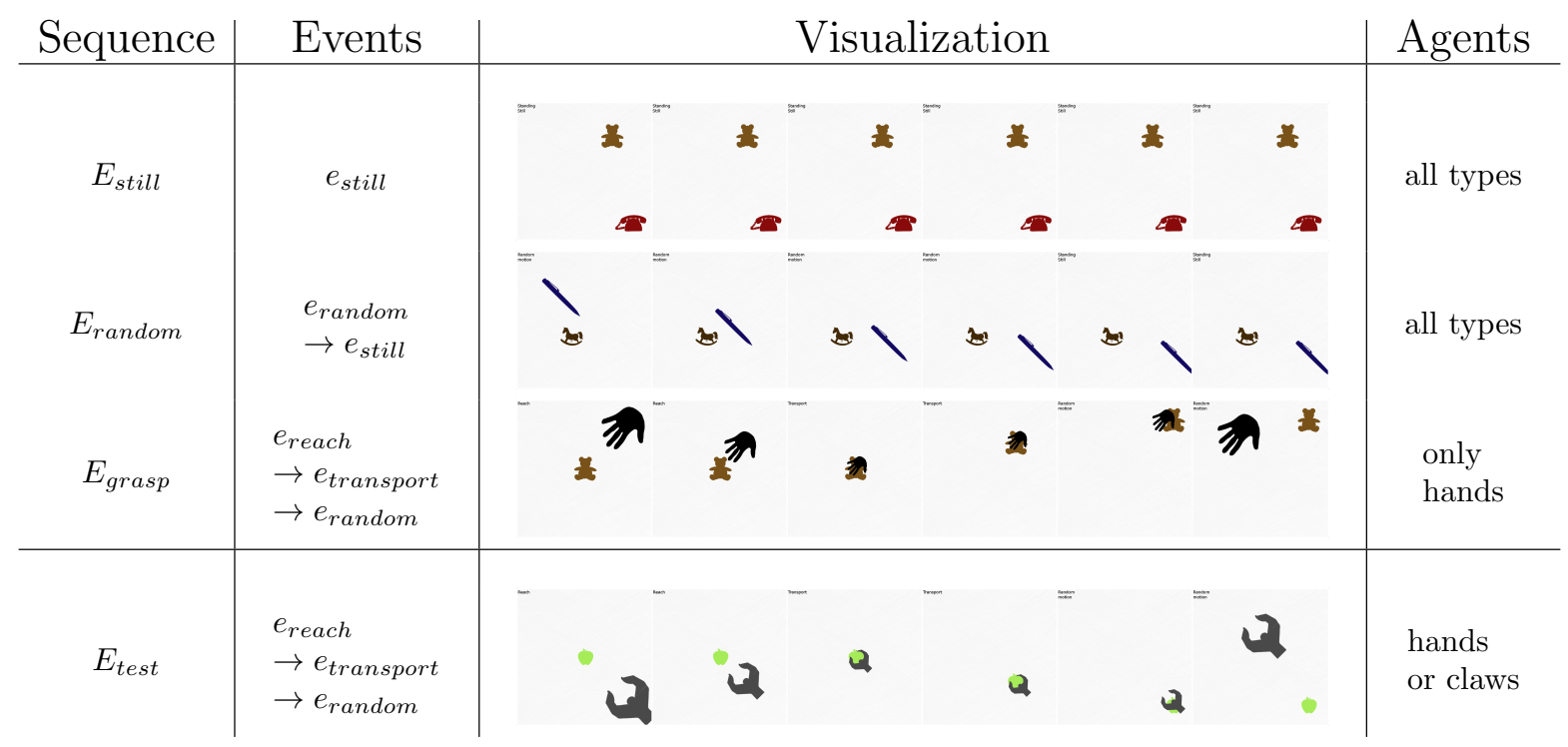

Figure 4: Event sequences, their individual events, and corresponding exemplar visualizations, rendered from a bird's-eye view (depth information can be deduced via entity size).

together to a randomly generated goal location with a randomly set, constant velocity.

By combining these events, three possible event sequences $E$ were trained (cf. Figure 4 ): In $E_{\text {grasp }}$, a hand agent reached for the patient $\left(e_{\text {reach }}\right)$, carried it to a certain location $\left(e_{\text {transport }}\right)$, and then let go of it and randomly moved away $\left(e_{\text {random }}\right)$. E $E_{\text {random }}$ started with a randomly directed motion event $\left(e_{\text {random }}\right)$ followed by both entities standing still $\left(e_{\text {still }}\right)$. $E_{\text {still }}$ showed both entities standing still $\left(e_{\text {still }}\right)$. For testing, we considered a testing sequence $E_{\text {test }}$, which is generally identical to $E_{\text {grasp }}$, but also allowed claw agents.

During supervised training, the system was informed about the type of event that currently unfolded. Each training epoch was composed of 100 event sequences, each uniformly randomly chosen and lasting between 50 and 150 time steps. Additionally, the eye fixation policy $\pi$ was randomly chosen and stayed fixed for every event sequence.

During testing, we tracked the system's gaze while the system was shown grasping sequences, mimicking actual 'experimental conditions' that investigated the occurrence of goal-anticipation in infants (Adam et al., 2016, 2017; Cannon \& Woodward, 2012; Kanakogi \& Itakura, 2011). In contrast to training, the system had to infer which event was currently observed using its learned event-predictive model components (cf. Section 4.2). Additionally, the system chose its gaze policy using active inference (cf. Section 4.3). Similar to Adam et al. (2016), we distinguished between two testing conditions: In the hand-condition, the system was shown a grasping sequence $E_{\text {test }}$ performed by a hand agent $\left(s^{a}=s_{\text {hand }}\right)$, similar to the ones shown in training. In the claw-condition, the same event sequence $E_{\text {test }}$ was shown being performed by a claw agent, which differed in its shape sensory signal $\left(s^{a}=s_{\text {claw }}\right)$. No model updates were performed during testing. Every test phase was composed of $10 E_{\text {test }}$ event sequences each for a hand and a claw agent. In order to assess whether goal-anticipatory gaze behavior emerges over the course of learning, we alternated between 


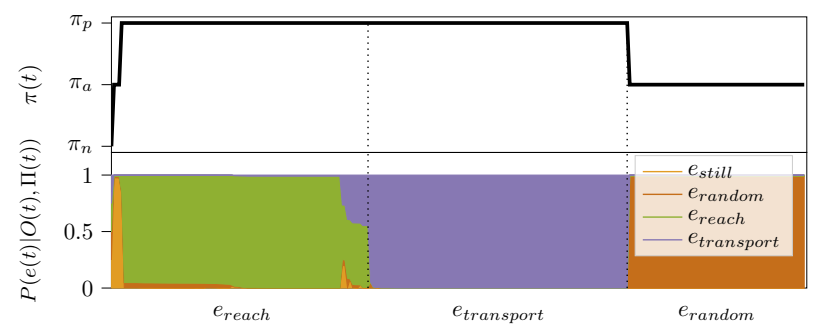

(a) hand agent

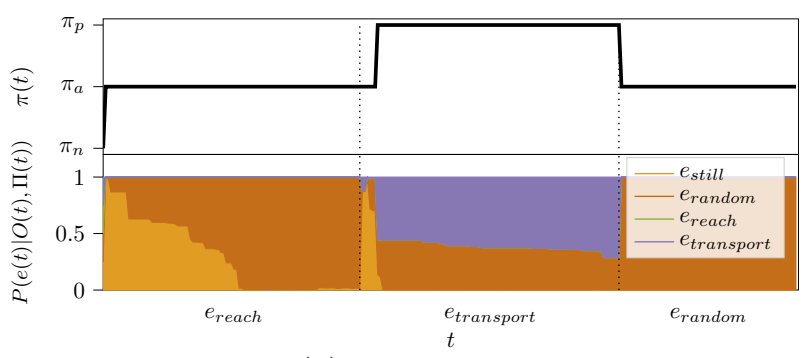

(b) claw agent

Figure 5: Exemplary event inference and policy inference after full training (30 epochs) over the course of one event sequence $E_{\text {test }}$ for a hand agent (a) and a claw agent (b). The top row in each sub-figure shows the active policy $\pi(t)$ over time $t$. The bottom row shows the inferred event probability estimates for the four possible events over time $t$. Event boundaries are marked by dotted lines.

training epochs and test phases.

\subsection{System behavior}

We focused on analyzing whether and under which conditions CAPRI generates goal-anticipatory gaze after learning. We provide further experiments on learning progressions, parameter dependencies, and ablations in the Supplementary Material sections 8.5-8.7. We trained CAPRI for 30 training epochs each followed by a test phase. We used the temporal horizon $\tau=1$ for policy inference. We ran this experiment 20 times with different, randomly chosen initial model weight and simulation parameter values. During the test phases, we mainly measured two quantities: (1) the internally estimated event-predictive probabilities $P\left(e_{i}(t) \mid O(t), \Pi(t)\right)$, to quantify the system's beliefs about the unfolding events. (2) the chosen gaze policy $\pi(t)$, to test whether the system activated $\pi_{p}$ (that is, <looking at the patient $>$ ) before the reaching event $e_{\text {reach }}$ ends, that is, whether the system starts to exhibit anticipatory gaze shifts for the hand agent and/or for the claw agent after having accumulated sufficient training experiences.

Figure 5 shows the inferred event probabilities for two exemplary event sequences $E_{\text {test }}$ for a fully trained system: (a) depicts the inferred event probabilities for a hand agent, (b) for a claw agent. During the beginning of a reaching movement of a hand, CAPRI inferred a high probability of standing still (i.e., $\left.P\left(e_{\text {still }}\right)\right)$. While accumulating more sensory observations, $P\left(e_{\text {still }}\right)$ decreased while $P\left(e_{\text {reach }}\right)$ sharply increased. After approximately 5 time steps, the system correctly assumed that a reaching event was unfolding with more than 95\% probability. As the hand got closer to the patient, the system started to anticipate that the reaching event will end soon, yielding a continuous decrease in $P\left(e_{\text {reach }}\right)$ and an increase in $P\left(e_{\text {transport }}\right)$. Once the hand touched the patient and started transporting it, $P\left(e_{\text {transport }}\right)$ sharply rose to approximately $100 \%$. Later on, when the hand let go of the patient and started a random motion, $P\left(e_{\text {random }}\right)$ quickly reached almost $100 \%$ probability.

The inferred event probabilities drastically differ when a claw agent is performing the same movement (Figure 5 (b)). While the probability for a standing still event continuously decreased during the first 
half of the reaching event, $P\left(e_{\text {random }}\right)$ increased and reached approximately $100 \%$. When the claw started transporting the object, CAPRI first incorrectly inferred that a standing still event was most likely, which then morphed into an approximately 50/50 guess in favor of $e_{\text {random }}$ and $e_{\text {transport }}$, progressively favoring $e_{\text {transport }}$ slightly. Once the claw let go of the patient and started a random motion, the system correctly inferred $P\left(e_{\text {random }}\right)$, quickly reaching almost $100 \%$ probability.

The differences in event inference also resulted in differences in gaze behavior for hand and claw agents. For hand agents, CAPRI started looking at the patient, i.e., it activated $\pi_{p}$, once it was certain that it was observing a reaching event (>90\% probability). Thus, for a hand agent we observe goal-anticipatory gaze shifts: the system looked at the reaching target before the target was reached by the agent. For a claw agent, the system kept tracking the agent, i.e. it activated $\pi_{a}$, during the whole reaching movement. Thus, for a claw agent, the system did not show goal-anticipatory gaze shifts.

Figure 6 shows the mean inferred event probability during the test phases for each of the four possible events $e_{\text {still }}, e_{\text {random }}, e_{\text {reach }}$, and $e_{\text {transport }}$, as a function of progressively more learning experience for hand agents (left-hand side) and claw agents (right-hand side) ${ }^{3}$. In all cases, CAPRI initially tended to infer $e_{\text {still }}$ and $e_{\text {random }}$ with high probability. Over the course of training, the system started to correctly infer progressively higher probabilities for the actual underlying events when the hand executed $e_{\text {reach }}, e_{\text {transport }}$, and $e_{\text {random }}$. In contrast, when CAPRI observed a claw agent, it incorrectly inferred $e_{\text {still }}$ and $e_{\text {random }}$ with much higher probability, even when $e_{\text {reach }}$ or $e_{\text {transport }}$ were shown. Proper event inference failed because the executing claw agent did not match the start condition learned for $e_{\text {reach }}$ and $e_{\text {transport }}$.

Figure 7a shows how the gaze behavior evolves with training experience. Here we measured at what time CAPRI on average activated the policy $\pi_{p}$ during an event sequence $E_{t e s t}$. Simulations where $\pi_{p}$ was not activated counted as looking at the patient at the end of the sequence. We computed the mean time of activating $\pi_{p}$ over simulations. When testing after very few training phases, the system did not look at the patient during the reaching event irrespective of hand or claw agent. From after the fourth training phase onwards, however, the system began to systematically activate $\pi_{p}$ in the beginning of the reaching event in $e_{\text {reach }}$ in the case of a hand agent. This goal-anticipatory gaze behavior was not shown by CAPRI for claw agents.

These results indicate that, once sufficiently trained on all four events and three event sequences, CAPRI generates gaze behavior that can be compared to that observed in 12-month-old infants (Adam et al., 2016). Figure $7 \mathrm{~b}$ shows the mean gaze arrival time for 12-month-olds watching movies of reaching movements performed by hand agents or claw agents (Adam et al., 2016). The movies started with a $500 \mathrm{~ms}$ still frame depicting an object, and then showed a hand or claw entering from the upper part of the screen (500-960 ms),

\footnotetext{
${ }^{3}$ We compute for every event $e_{i} \in E_{t e s t}$ and every simulation $s_{k}$ the mean probability as $\bar{P}_{s_{k}}\left(e_{j} \mid O\left(e_{i}\right), \Pi\left(e_{i}\right)\right)=$ $\sum_{t=t_{s}}^{t_{e}} P\left(e_{j}(t) \mid O(t), \Pi(t)\right) \frac{1}{t_{e}-t_{s}}$, with $t_{s}$ marking the start of $e_{i}$ and $t_{e}$ marking its end and for all $e_{j} \in$ $\left\{e_{\text {still }}, e_{\text {random }}, e_{\text {reach }}, e_{\text {transport }}\right\} . \bar{P}_{s_{k}}\left(e_{j} \mid O\left(e_{i}\right), \Pi\left(e_{i}\right)\right)$ can be visualized when looking at Figure 5 . If one takes the mean probabilities during one event $e_{i}$, bounded by dotted lines, one gets the estimate of $\bar{P}_{s_{k}}\left(e_{j} \mid e_{i}, O, \Pi\right)$. This probability is computed for every test phase of every simulation and Figure 6 shows $\bar{P}\left(e_{j} \mid e_{i}, O, \Pi\right)$ averaged over all simulations $s_{k}$.
} 


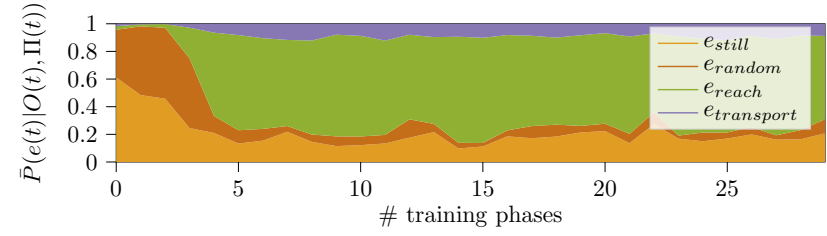

(a) Event probabilities for $e_{\text {reach }}$ and hand agents

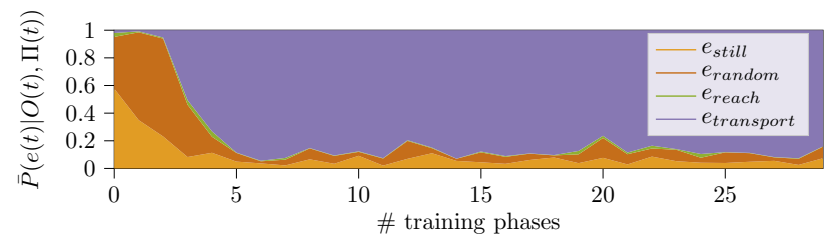

(c) Event probabilities for $e_{\text {transport }}$ and hand agents

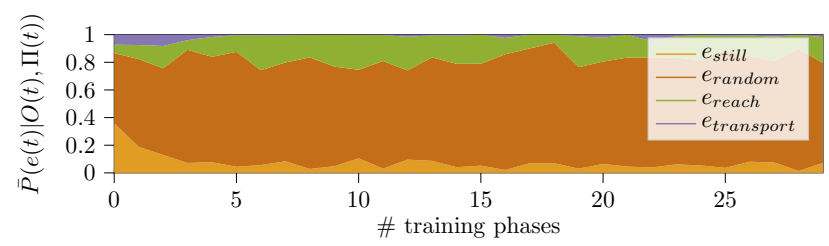

(e) Event probabilities for $e_{\text {random }}$ and hand agents

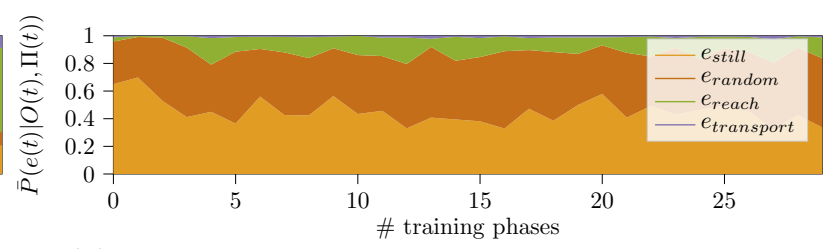

(b) Event probabilities for $e_{\text {reach }}$ and claw agents

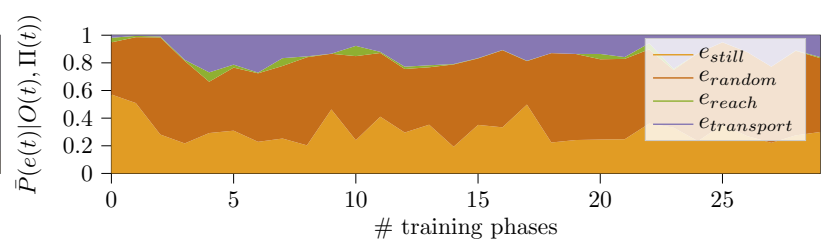

(d) Event probabilities for $e_{\text {transport }}$ and claw agents

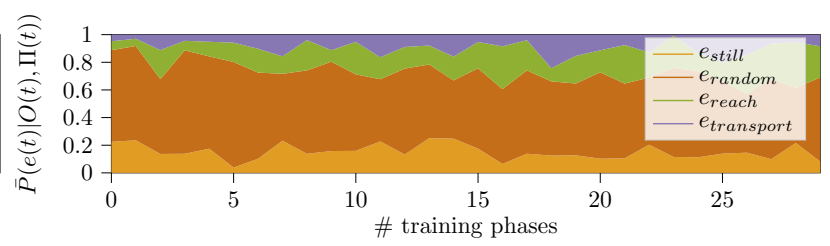

(f) Event probabilities for $e_{\text {random }}$ and claw agents

Figure 6: Mean inferred event probabilities $\bar{P}\left(e_{i}(t) \mid O(t), \Pi(t)\right)$ for $e_{i} \in\left\{e_{\text {still }}, e_{\text {random }}, e_{\text {reach }}, e_{\text {transport }}\right\}$ during $E_{\text {test }}$ over the course of learning for hand agents (a,c,e) and claw agents (b,d,f). (a) and (b) show the event probabilities during the presentation of $e_{\text {reach }}$, (c) and (d) during $e_{\text {transport }}$, and (e) and (f) during $e_{\text {random. }}$.

pausing shortly (960-1800 ms), and then reaching for the target object (1800-2920 ms). To measure the gaze arrival times, two areas of interest (AOI) were created to cover the agent and the target object for each movie. Mean gaze arrival times were calculated by subtracting the time when the agent entered the target AOI from the time of the first target AOI fixation. Thus, a negative gaze arrival time corresponds to goal-anticipatory gaze. As shown in Figure 7b, when the infants observed reaching movies done by hands, they tended to look at the target AOI before the hand arrived. For the claw, the infants tended to first look at the target AOI upon arrival of the agent. Similar behavior developed in CAPRI (compare results in Figures 7a and 7b), indicating that infants' goal-anticipatory gaze shifts may rely on processes and principles similar to those implemented in CAPRI.

\subsection{Discussion of results}

Over the course of training, CAPRI tended to improve its ability to correctly infer the event probabilities for a hand agent. For claw agents, the system produced incorrect inferences. This is mainly due to the system learning that reaching and transporting events are typically performed by hand-agents, which can be recognized from their observed shape $\left(s_{a}=s_{\text {hand }}\right)$. Agents with a different shape $s_{a}$, such as claw agents, are not known to execute reaching- and transporting events. In conjunction with the active inference mechanism, 


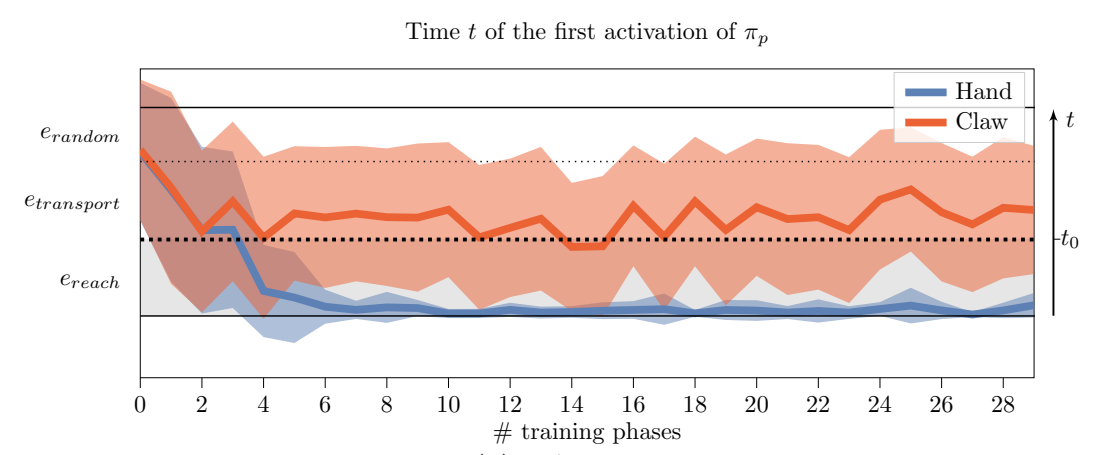

(a) CAPRI

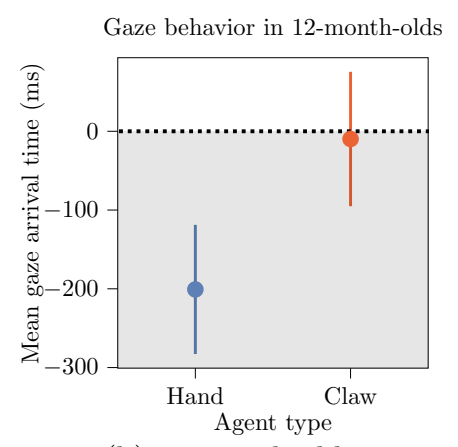

(b) 12-month-olds

Figure 7: Analyzing anticipatory gaze for reaching movements. (a) shows the gaze behavior for CAPRI over the course of learning. We plot the mean time $t$ during an event sequence $E_{\text {test }}$ when the system first activated policy $\pi_{p}$, which corresponds to looking at the patient. Event boundaries are marked by dotted horizontal black lines with $t_{0}$ marking the agent's arrival at the patient. Shaded areas show the standard deviation across simulations. (b) shows the anticipatory gaze shifts in ms for 12-month-old infants taken from Adam et al. (2016). The dotted horizontal line marks the agent's arrival at the patient $(t=0)$. Error bars show the standard error. In both plots a data point in the gray area, below the dotted line denoting the agent's arrival at the patient, marks an anticipatory gaze.

CAPRI thus begins to show goal-anticipatory gaze behavior over the course of training when a hand agent is observed, but not when a claw agent is observed. Similarly, 12-month-old infants, who already have some experience with self-performed and observed human grasping, tend to shift their gaze to the goal of reaching before there is contact between hand and target, but they do not do so when a claw agent is observed (Adam et al., 2016).

Please note that CAPRI was not trained nor pre-programmed to generate goal-anticipatory gaze behavior. Instead, the behavior emerged purely from the system estimating the ongoing event via Equation 1 and choosing its actions by means of active inference, which aims at decreasing expected uncertainty about the ongoing event and the upcoming event boundaries according to Equation 10. During the beginning of training, CAPRI did not show goal-anticipatory gaze shifts during $E_{\text {test }}$ because the system (i) did not recognize the underlying event, inferring a low probability for $e_{\text {reach }}$, and (ii) had not yet learned which type of policy could be expected to result in a decrease in future uncertainty ${ }^{4}$. At this point $e_{\text {random }}$ or $e_{\text {still }}$ were inferred, during which CAPRI could minimize uncertainty best by looking at the agent, that is, choosing gaze policy $\pi_{a}$. Once the event-predictive models were sufficiently precise and it became known that fixating the target object served to decrease uncertainty for both, the reaching event and the exact time when the next event boundary would occur, CAPRI generated goal-anticipatory gaze shifts. In particular, for a reaching event $e_{\text {reach }}$, the system could minimize uncertainty best by fixating the patient, that is, choosing gaze policy $\pi_{p}$, because this resulted in less noisy information about the patient's position, which informed the system about where reaching would end. As a result, goal-anticipatory gaze behavior emerged over the course of training when observing a hand reaching for an object.

\footnotetext{
${ }^{4}$ We further investigate how the experience with $E_{\text {grasp }}$ in particular affects the goal-anticipatory gaze in an additional experiment in Section 8.5 of the Supplementary Material.
} 
We propose that similar processes are involved when infants exhibit goal-anticipatory gaze behavior (e.g., Elsner \& Adam, 2021). For most events involving a moving agent, tracking the agent can give the most information about the future, such as the agent's future position and velocity. However, the end of a reaching event can be predicted best when the position of the target object is known, which can be estimated best when looking at the target before the reaching agent arrives at the target. Fittingly, goal-anticipatory gaze behavior was found when adults performed, and when they observed another person perform, a block-stacking task (Flanagan \& Johansson, 2003). For observed reaching actions, infants' anticipatory gaze depends on their action experience and on the familiarity of the agent, starting from about 7 months for human hands, when infants typically achieve the developmental milestone of visually-guided grasping (Adam et al., 2016; Falck-Ytter et al., 2006; Kanakogi \& Itakura, 2011). In CAPRI, goal-anticipatory gaze depends on whether the reaching event is recognized or not. We assume that infants likewise associate a perceived start condition of visuospatial features of a hand together with a graspable object with the encoding of an event $e_{\text {reach }}$, and, as a result, only activate their generative model for reaching when sufficient (active or observed) grasping experience has been acquired and when sufficiently many relevant indicators are perceived. Based on their stored event schemas, infants can then create a forward model of the agent's future trajectory and the event's typical end condition (Elsner \& Adam, 2021). When the start condition contains an unfamiliar agent, such as a mechanical claw, the event schemata necessary for eliciting goal-anticipatory gaze behavior are typically not activated.

Thus, according to CAPRI, 12-month-olds track the reaching motion of unfamiliar agents because they fail to recognize the start of a reaching event and, as a result, cannot tap on their stored event knowledge in order to anticipate how this event will end. An alternative explanation for the findings could be that the infants found it difficult to disengage their gaze from the interesting unfamiliar agent, which in turn made it more difficult for them to shift their gaze to the goal object ahead of time. However, recent research indicates that infants typically do not show longer looking-times to unfamiliar claws compared to familiar hands in simple grasping events (e.g., Adam et al., 2016; Adam \& Elsner, 2020). Hence, it is unlikely that this gaze behavior could solely be explained by general mechanisms like a limited capacity for stimulus disengagement.

While a comparable gaze behavior is shown by CAPRI and the 12-month-old infants, there are numerical differences worth discussing. For example, the gaze shifts towards the target object occurred later in infants than in CAPRI. Clearly though, the experimental results of CAPRI and the 12-month-olds are not quantitatively comparable, because Adam et al. (2016) used different stimuli and an AOI-based measure for gaze recording. Moreover, our scenario is strongly simplified compared to the learning and testing of infants. CAPRI sees reaching movements exactly how they occur during training, while infants see the particular reaching movement for the first time at the start of the experiment, and can further process the movement (and relate it to their previously gained action experience) across about 12 repeated presentations. Besides that, CAPRI always observes the shown simulation, while infants can also avert their gaze from the screen or close their eyes. However, the aim of our study was not to exactly replicate the infant data, but to analyze 
the functional foundations of anticipatory, event-predictive gaze behavior. Generally, CAPRI should achieve quantitatively similar results in richer simulation environments. Additional model evaluations provided in the supplementary material largely support this claim: Decreasing the percentage of $e_{\text {reach }}$ events encountered during training (Section 8.5) or increasing the difficulty of recognizing the hand (Section 8.7) indeed delay the development of anticipatory gaze behavior. Along similar lines, we assume that a richer simulation environment would increase event inference difficulty and, thus, should likely delay the anticipatory gaze behavior of CAPRI, possibly matching the infants' data even more exactly.

\section{Discussion and future work}

In this paper, we have proposed how goal-anticipatory gaze shifts in infants can emerge. We introduced and implemented CAPRI, a computational and algorithmic model that learns generative, schematic event encodings, which predict the dynamics of an event in a probabilistic manner and also predicts distributions modeling the typical conditions at the start and end of an event. CAPRI tries to constantly infer which one of the previously learned events is currently unfolding by iteratively inferring event likelihood distributions dependent on its learned event-predictive schemata and the incoming sensory observations. Besides inferring events, CAPRI inferred its action policy aiming solely at minimizing expected future uncertainty, which was equated with the entropy of the current event distribution and the expected next possible event boundary distributions. As a result, CAPRI can be understood as a modified hidden Markov model, which processes dynamic Bayesian likelihood estimates as done recently elsewhere (e.g., Franklin et al., 2020), but which additionally infers actions by means of event-predictive active inference.

We tested CAPRI in a simple agent-patient interaction scenario where policies corresponded to different gaze strategies. The system showed anticipatory gaze behavior similar to the goal-anticipatory gaze shifts previously found in eye-tracking studies in infants (Adam et al., 2016; Cannon \& Woodward, 2012; Kanakogi \& Itakura, 2011). When a hand agent was performing a reaching action that had been observed during training, CAPRI looked at the target before reaching was complete. These goal-anticipatory gaze shifts could not be observed when CAPRI only had little experience in reaching. Similarly, 12-month-old infants look at the target of a hand-reaching movement before the reaching is concluded (e.g., Adam et al., 2016), while infants younger than 6 months, who are believed to have little to no experience in reaching, do not. Moreover, when CAPRI observed a reaching event with an unfamiliar claw agent, which never performed reaching actions during training, our system did not identify reaching and preferred to track the agent over the course of the event. Similarly, when 12-month-olds observe a mechanical claw reaching for an object, they tend to perform tracking gaze (Adam et al., 2016).

Note, that none of these effects were explicitly programmed into the system. Both the structure of event-representations and the minimization of predicted uncertainty have been proposed as key mechanisms of cognition over the last decades by various theories, such as the Theory of Event Coding (Hommel et al., 
2001), Event Segmentation Theory (Zacks et al., 2007), Predictive Coding (Rao \& Ballard, 1999), and the Free Energy Principle (Friston, 2010). Showing how behavioral effects can emerge based on these theories further supports the proposition to merge these theories for developing a unifying theory of cognition (Butz, 2016) and allows for an integrative theoretical perspective on infants' anticipatory gaze behavior (Elsner \& Adam, 2021). Goal-anticipatory gaze behavior essentially emerged from the structure in which events were encoded during the training and from the objective to invoke behavioral policies, that is, eye fixation targets, that are expected to minimize predicted uncertainty in the near future.

Nonetheless, various aspects and design considerations demand future modeling work. First, in the current implementation, we provided supervised information about the event that is currently observed during training. Additionally, we specified for each event which entity takes the role of the agent and the patient. Clearly this is unrealistic. Because we aimed at investigating whether goal-anticipatory gaze behavior can emerge at all from learned event schemata in combination with active inference processes, we omitted the challenge of automatically individualizing event schemata models and the roles of the involved entities from the continuous stream of sensorimotor information. Nevertheless, in future work we want to investigate whether we can find the same effects when event schemata are learned in a fully self-supervised manner. There are different methods for the self-supervised extraction of event models from the continuous stream of sensorimotor information, such as surprise-based segmentation (Gumbsch et al., 2019; Reynolds, Zacks, \& Braver, 2007) or retrospective gradient-based hidden state inference (Butz et al., 2019). Furthermore, the role of an entity with a specific shape could be inferred from the sensorimotor data, for example via the detection of "mover"-events, i.e., events in which the movement of one entity leads to the movement of another entity (Ullman, Harari, \& Dorfman, 2012). Further investigations are required to identify which type of segmentation methods, or combinations of such methods, are most suitable.

Second, CAPRI chooses actions to minimize predicted uncertainty over a fixed temporal horizon $\tau$ into the future. This is sufficient for the investigated simple scenario. However, the effects of goal-anticipation might emerge even more strongly when the system attempts to predict the future only when it is certain about the present. Therefore, our further research will consider a more flexible temporal horizon of predictions based on estimated uncertainty. This may strengthen the effects of goal-anticipations found in the current study and may enable the emergence of goal-anticipatory gaze shifts in more complicated simulations and without an explicit limit on the temporal horizon $\tau$.

Third, CAPRI learns from own action experiences, utilizing those experiences for inferring subsequently observed action events. By including relative encodings between agent and patient, we somewhat sidestepped the perspective-taking challenge (Moll \& Meltzoff, 2011; Tversky \& Hard, 2009), as has been done elsewhere (Schrodt \& Butz, 2016). However, infant studies obtained comparable results for stimuli presented from various spatial perspectives (Adam et al., 2016, 2017), calling the relevance of this factor for anticipatory gaze behavior into question. Moreover, we somewhat ignored the fact that motor signals are available when executing particular actions, which probably support goal-anticipatory gaze behavior via direct matching 
when observing others performing a similar action (e.g. Gredebäck \& Falck-Ytter, 2015). In future modeling work, we intend to enhance CAPRI to address these challenges in further detail.

Besides such system expansions and modifications, we intend to model other effects of goal-anticipation in infants. For example, it has been shown that 11-month-old infants perform a goal-anticipatory gaze shift for mechanical claws when the claw shows cues of agency, such as self-propelled motion and a salient action-effect (Adam et al., 2017). Additionally, EEG studies found that from 9 months onwards, infants show predictive sensorimotor-cortex activity for actions performed by various agents such as a hand, a mechanical claw, or a self-propelled toy (Southgate \& Begus, 2013). After the infants had been familiarised with videos in which the agent grasped and transported the object to a different location, or in which the object moved to a new location by itself, predictive motor activity was found in the infants' brains even when only a still frame of the start of the action event was presented. Thus, instead of directly conditioning on the agent's appearance, more general agency cues, such as self-propelled movement, may be encoded in the likelihood distributions of our event schemata. The presence of such agency cues adds visuospatial features that may help to identify goal-directed events, such as reaching, thereby enabling the generation of goal-anticipatory gaze shifts.

Moreover, experimental data with adults demands further model-based, computational explanations. Anticipatory gaze shifts have been investigated in various scenarios, including complex object interactions (Belardinelli, Barabas, Himmelbach, \& Butz, 2016; Belardinelli, Stepper, \& Butz, 2016; Hayhoe, Shrivastava, Mruczek, \& Pelz, 2003). Moreover, in anticipatory cross-modal interactions, the future temporal horizon appears to be event-oriented and was shown to depend on predictable forthcoming event uncertainties (Belardinelli, Lohmann, Farnè, \& Butz, 2018; Lohmann, Belardinelli, \& Butz, 2019). We expect that our event-generative inference model is applicable to the observed data patterns, because similar event-generative encodings and inference processes should be at play.

Lastly, it needs to be noted that for now, CAPRI only focuses on goal anticipation measured via anticipatory gaze shifts. Therefore, our results might not necessarily be applicable to post-hoc measures such as looking-times (e.g., Woodward, 1998) or to other predictive processes such as EEG activity (e.g., Southgate \& Begus, 2013). As of now, the relations between these different measures are still widely unknown, and more research needs to be conducted to fully understand how these measures do or do not capture the same processes regarding infants' processing of goal-directed actions.

To conclude, let us consider the question which type of representations and inference processes may give rise to goal-anticipatory gaze behavior in infants. It has been proposed that infants learn flexible goal representations for each action event (Cannon \& Woodward, 2012). An alternative explanation suggests that infants rely on trajectory-based information when estimating how an observed movement will end (Ganglmayer, Attig, Daum, \& Paulus, 2019). Along similar lines, goal-anticipation in infants was previously modeled in a complex robotic reaching scenario using closed-loop sensorimotor forward simulations (Copete, Nagai, \& Asada, 2016). There seems to be experimental evidence that supports both flexible goal-representations and trajectory-based models (Cannon \& Woodward, 2012; Ganglmayer et al., 2019). Our modeling results 
suggest that both types of representation are involved. Event dynamics models encode how the observation will change during one event over a short period of time, essentially predicting the exact trajectory of an ongoing motion. However, inferring the goal of an event using a closed-loop simulation is costly and prone to accumulate error. The event-generative encoding of end conditions enables their direct anticipation, but this requires previous (active or passive) experience with the observed action (Elsner \& Adam, 2021). Similarly, start-condition encodings enable hierarchical, closed-loop simulations of event successions, without the simulation of detailed event dynamics. As a result, these types of representation can be directly accessed to infer goal-anticipatory gaze behavior and thus to plan and reason on deeper, event-predictive levels. The available developmental-psychology research and the CAPRI model we have introduced in this paper suggest that we learn the compositional basis of such deeper cognitive abilities via the observation of action events during the first year of life.

\section{Acknowledgments}

This research was funded by the German Research Foundation (DFG) within Priority-Program SPP 2134 project "Development of the agentive self" (BU 1335/11-1, EL 253/8-1). The authors thank the International Max Planck Research School for Intelligent Systems (IMPRS-IS) for supporting Christian Gumbsch. Martin Butz is a member of the Machine Learning Cluster of Excellence, EXC number 2064/1 - Project number 390727645 .

\section{References}

Adam, M., \& Elsner, B. (2018). Action effects foster 11-month-olds' prediction of action goals for a non-human agent. Infant Behavior and Development, 53, 49-55. doi: 10.1016/j.infbeh.2018.09.002

Adam, M., \& Elsner, B. (2020). The impact of salient action effects on 6-, 7-, and 11-month-olds' goal-predictive gaze shifts for a human grasping action. PLOS ONE, 15(10), 1-18. doi: 10.1371/journal.pone.0240165

Adam, M., Reitenbach, I., \& Elsner, B. (2017). Agency cues and 11-month-olds' and adults' anticipation of action goals. Cognitive Development, 43, 37-48. doi: 10.1016/j.cogdev.2017.02.008

Adam, M., Reitenbach, I., Papenmeier, F., Gredebäck, G., Elsner, C., \& Elsner, B. (2016). Goal saliency boosts infants' action prediction for human manual actions, but not for mechanical claws. Infant Behavior and Development, 44, 29-37. doi: 10.1016/j.infbeh.2016.05.001

Baldwin, D. A., \& Kosie, J. E. (2021). How does the mind render streaming experience as events? Topics in Cognitive Science, 13(1), 79-105. doi: https://doi.org/10.1111/tops.12502

Belardinelli, A., Barabas, M., Himmelbach, M., \& Butz, M. V. (2016). Anticipatory eye fixations reveal tool knowledge for tool interaction. Experimental Brain Research, 234(8), 2415-2431. doi: 10.1007/ s00221-016-4646-0 
Belardinelli, A., Lohmann, J., Farnè, A., \& Butz, M. V. (2018). Mental space maps into the future. Cognition, 176, 65-73. doi: 10.1016/j.cognition.2018.03.007

Belardinelli, A., Stepper, M. Y., \& Butz, M. V. (2016). It's in the eyes: Planning precise manual actions before execution. Journal of Vision, 16(1), 18-18. doi: 10.1167/16.1.18

Biro, S. (2013). The role of the efficiency of novel actions in infants' goal anticipation. Journal of Experimental Child Psychology, 116(2), 415-427. doi: 10.1016/j.jecp.2012.09.011

Bishop, C. M. (2006). Pattern recognition and machine learning (information science and statistics). Berlin, Heidelberg: Springer-Verlag.

Botvinick, M., \& Toussaint, M. (2012). Planning as inference. Trends in Cognitive Sciences, 16(10), 485 488. doi: $10.1016 /$ j.tics.2012.08.006

Butz, M. V. (2016). Towards a unified sub-symbolic computational theory of cognition. Frontiers in Psychology, 7(925). doi: 10.3389/fpsyg.2016.00925

Butz, M. V. (2017). Which structures are out there. In T. K. Metzinger \& W. Wiese (Eds.), Philosophy and predictive processing (chap. 8). Frankfurt am Main: MIND Group. doi: 10.25358/openscience-631

Butz, M. V., Achimova, A., Bilkey, D., \& Knott, A. (2021). Event-predictive cognition: A root for conceptual human thought. Topics in Cognitive Science, 13(1), 10-24. doi: https://doi.org/10.1111/tops.12522

Butz, M. V., Bilkey, D., Humaidan, D., Knott, A., \& Otte, S. (2019). Learning, planning, and control in a monolithic neural event inference architecture. Neural Networks, 117, 135-144. doi: 10.1016/ j.neunet.2019.05.001

Cannon, E. N., \& Woodward, A. L. (2012). Infants generate goal-based action predictions. Developmental Science, 15(2), 292-298. doi: 10.1111/j.1467-7687.2011.01127.x

Cannon, E. N., Woodward, A. L., Gredebäck, G., von Hofsten, C., \& Turek, C. (2012). Action production influences 12-month-old infants' attention to others' actions. Developmental Science, 15(1), 35-42. doi: https://doi.org/10.1111/j.1467-7687.2011.01095.x

Cooper, R. P. (2021). Action production and event perception as routine sequential behaviors. Topics in Cognitive Science, 13(1), 63-78. doi: https://doi.org/10.1111/tops.12462

Copete, J. L., Nagai, Y., \& Asada, M. (2016). Motor development facilitates the prediction of others' actions through sensorimotor predictive learning. In 2016 joint ieee international conference on development and learning and epigenetic robotics (icdl-epirob) (p. 223-229). doi: 10.1109/DEVLRN.2016.7846823

Daum, M., Attig, M., Gunawan, R., Prinz, W., \& Gredebäck, G. (2012). Actions seen through babies' eyes: A dissociation between looking time and predictive gaze. Frontiers in Psychology, 3, 370. doi: 10.3389 /fpsyg. 2012.00370

Elsner, B., \& Adam, M. (2021). Infants' goal prediction for simple action events: The role of experience and agency cues. Topics in Cognitive Science, 13(1), 45-62. doi: https://doi.org/10.1111/tops.12494

Elsner, B., \& Hommel, B. (2001). Effect anticipation and action control. Journal of Experimental Psychology: Human Perception and Performance, 27(1), 229-240. doi: 10.1037//0096-1523.27.1.229 
Falck-Ytter, T., Gredebäck, G., \& von Hofsten, C. (2006). Infants predict other people's action goals. Nature Neuroscience, 9(7), 878-879. doi: 10.1038/nn1729

Fantz, R. L. (1958). Pattern vision in young infants. The Psychological Record, 8, 43-47. doi: 10.1007/ BF03393306

Flanagan, J. R., \& Johansson, R. S. (2003). Action plans used in action observation. Nature, 424(6950), 769-771. doi: 10.1038/nature01861

Franklin, N. T., Norman, K. A., Ranganath, C., Zacks, J. M., \& Gershman, S. J. (2020). Structured event memory: A neuro-symbolic model of event cognition. Psychological Review, 127(3), 327-361. doi: $10.1037 /$ rev0000177

Friston, K. (2010). The free-energy principle: a unified brain theory? Nature Reviews Neuroscience, 11(2), 127-138. doi: 10.1038/nrn2787

Friston, K., FitzGerald, T., Rigoli, F., Schwartenbeck, P., O’Doherty, J., \& Pezzulo, G. (2016). Active inference and learning. Neuroscience \& Biobehavioral Reviews, 68, 862-879. doi: https://doi.org/ 10.1016/j.neubiorev.2016.06.022

Friston, K., Rigoli, F., Ognibene, D., Mathys, C., FitzGerald, T., \& Pezzulo, G. (2015). Active inference and epistemic value. Cognitive Neuroscience, 6, 187-214. doi: 10.1080/17588928.2015.1020053

Gallese, V., Fadiga, L., Fogassi, L., \& Rizzolatti, G. (1996). Action recognition in the premotor cortex. Brain, 119(2), 593-609. doi: 10.1093/brain/119.2.593

Ganglmayer, K., Attig, M., Daum, M. M., \& Paulus, M. (2019). Infants' perception of goal-directed actions: A multi-lab replication reveals that infants anticipate paths and not goals. Infant Behavior and Development, 57, 101340. doi: https://doi.org/10.1016/j.infbeh.2019.101340

Gredebäck, G., \& Falck-Ytter, T. (2015). Eye movements during action observation. Perspectives on Psychological Science, 10(5), 591-598. doi: 10.1177/1745691615589103

Gredebäck, G., Johnson, S., \& von Hofsten, C. (2010). Eye tracking in infancy research. Developmental Neuropsychology, 35(1), 1-19. doi: 10.1080/87565640903325758

Gredebäck, G., \& Melinder, A. (2010). Infants' understanding of everyday social interactions: A dual process account. Cognition, 114 (2), 197-206. doi: 10.1016/j.cognition.2009.09.004

Gredebäck, G., Stasiewicz, D., Falck-Ytter, T., Rosander, K., \& von Hofsten, C. (2009). Action type and goal type modulate goal-directed gaze shifts in 14-month-old infants. Developmental Psychology, 45(4), 1190-1194. doi: 10.1037/a0015667

Gumbsch, C., Butz, M. V., \& Martius, G. (2019). Autonomous identification and goal-directed invocation of event-predictive behavioral primitives. IEEE Transactions on Cognitive and Developmental Systems. doi: 10.1109/TCDS.2019.2925890

Hayhoe, M. M., Shrivastava, A., Mruczek, R., \& Pelz, J. B. (2003). Visual memory and motor planning in a natural task. Journal of Vision, 3(1), 49-63. doi: 10:1167/3.1.6

Hommel, B. (2009). Action control according to tec (theory of event coding). Psychological Research PRPF, 
73 (4), 512-526. doi: 10.1007/s00426-009-0234-2

Hommel, B. (2015). The theory of event coding (tec) as embodied-cognition framework. Frontiers in Psychology, 6, 1318. doi: 10.3389/fpsyg.2015.01318

Hommel, B., Müsseler, J., Aschersleben, G., \& Prinz, W. (2001). The theory of event coding (tec): A framework for perception and action planning. Behavioral and Brain Sciences, 24(5), 849-878. doi: $10.1017 / \mathrm{s} 0140525 \times 01000103$

Kanakogi, Y., \& Itakura, S. (2011). Developmental correspondence between action prediction and motor ability in early infancy. Nature Communications, 2, 341. doi: 10.1038/ncomms1342

Knill, D. C., \& Pouget, A. (2004). The bayesian brain: the role of uncertainty in neural coding and computation. Trends in Neurosciences, 27(12), 712-719. doi: https://doi.org/10.1016/j.tins.2004.10.007

Krogh-Jespersen, S., \& Woodward, A. L. (2014). Making smart social judgments takes time: Infants' recruitment of goal information when generating action predictions. PloS one, 9(5), e98085. doi: 10.1371/journal.pone.0098085

Kuperberg, G. R. (2021). Tea with milk? a hierarchical generative framework of sequential event comprehension. Topics in Cognitive Science, 13(1), 256-298. doi: https://doi.org/10.1111/tops.12518

Lohmann, J., Belardinelli, A., \& Butz, M. V. (2019). Hands ahead in mind and motion: Active inference in peripersonal hand space. Vision, 3(2), 15. doi: 10.3390/vision3020015

Moll, H., \& Meltzoff, A. (2011). Perspective-taking and its foundation in joint attention. In (p. 286-304). doi: 10.1093/acprof:oso/9780199692040.003.0016

Radvansky, G. A., \& Zacks, J. M. (2014). Event cognition. Oxford University Press. doi: 10.1093/acprof:oso/ 9780199898138.001.0001

Rao, R. P., \& Ballard, D. H. (1999). Predictive coding in the visual cortex: a functional interpretation of some extra-classical receptive-field effects. Nature Neuroscience, 2(1), 79. doi: 10.1038/4580

Reynolds, J. R., Zacks, J. M., \& Braver, T. S. (2007). A computational model of event segmentation from perceptual prediction. Cognitive Science, 31(4), 613-643. doi: 10.1080/15326900701399913

Rizzolatti, G., Fadiga, L., Gallese, V., \& Fogassi, L. (1996). Premotor cortex and the recognition of motor actions. Cognitive Brain Research, 3(2), 131-141. doi: 10.1016/0926-6410(95)00038-0

Rizzolatti, G., Fogassi, L., \& Gallese, V. (2001). Neurophysiological mechanisms underlying the understanding and imitation of action. Nature reviews Neuroscience, 2(9), 661-670. doi: 10.1038/35090060

Schrodt, F., \& Butz, M. V. (2016). Just imagine! learning to emulate and infer actions with a stochastic generative architecture. Frontiers in Robotics and AI, 3, 5. doi: 10.3389/frobt.2016.00005

Southgate, V., \& Begus, K. (2013). Motor activation during the prediction of nonexecutable actions in infants. Psychological Science, 24(6), 828-835. doi: 10.1177/0956797612459766

Stawarczyk, D., Bezdek, M. A., \& Zacks, J. M. (2021). Event representations and predictive processing: The role of the midline default network core. Topics in Cognitive Science, 13(1), 164-186. doi: https://doi.org/10.1111/tops.12450 
Tversky, B., \& Hard, B. M. (2009). Embodied and disembodied cognition: Spatial perspective-taking. Cognition, 110(1), 124-129. doi: https://doi.org/10.1016/j.cognition.2008.10.008

Ullman, S., Harari, D., \& Dorfman, N. (2012). From simple innate biases to complex visual concepts. Proceedings of the National Academy of Sciences, 109(44), 18215-18220. doi: 10.1073/pnas.1207690109

Woodward, A. L. (1998). Infants selectively encode the goal object of an actor's reach. Cognition, 69(1), 1-34. doi: 10.1016/S0010-0277(98)00058-4

Zacks, J. M., Speer, N. K., Swallow, K. M., Braver, T. S., \& Reynolds, J. R. (2007). Event perception: a mind-brain perspective. Psychological Bulletin, 133(2), 273-293. doi: 10.1037/0033-2909.133.2.273

Zacks, J. M., \& Swallow, K. M. (2007). Event segmentation. Current Directions in Psychological Science, 16(2), 80-84. doi: 10.1111/j.1467-8721.2007.00480.x

Zacks, J. M., \& Tversky, B. (2001). Event structure in perception and conception. Psychological Bulletin, 127(1), 3-21. doi: 10.1037/0033-2909.127.1.3 


\section{Supplementary Material}

\subsection{Pseudocode}

Here we provide pseudocode of CAPRI. A Python implementation can be found at https://github.com/ CognitiveModeling/CAPRI.

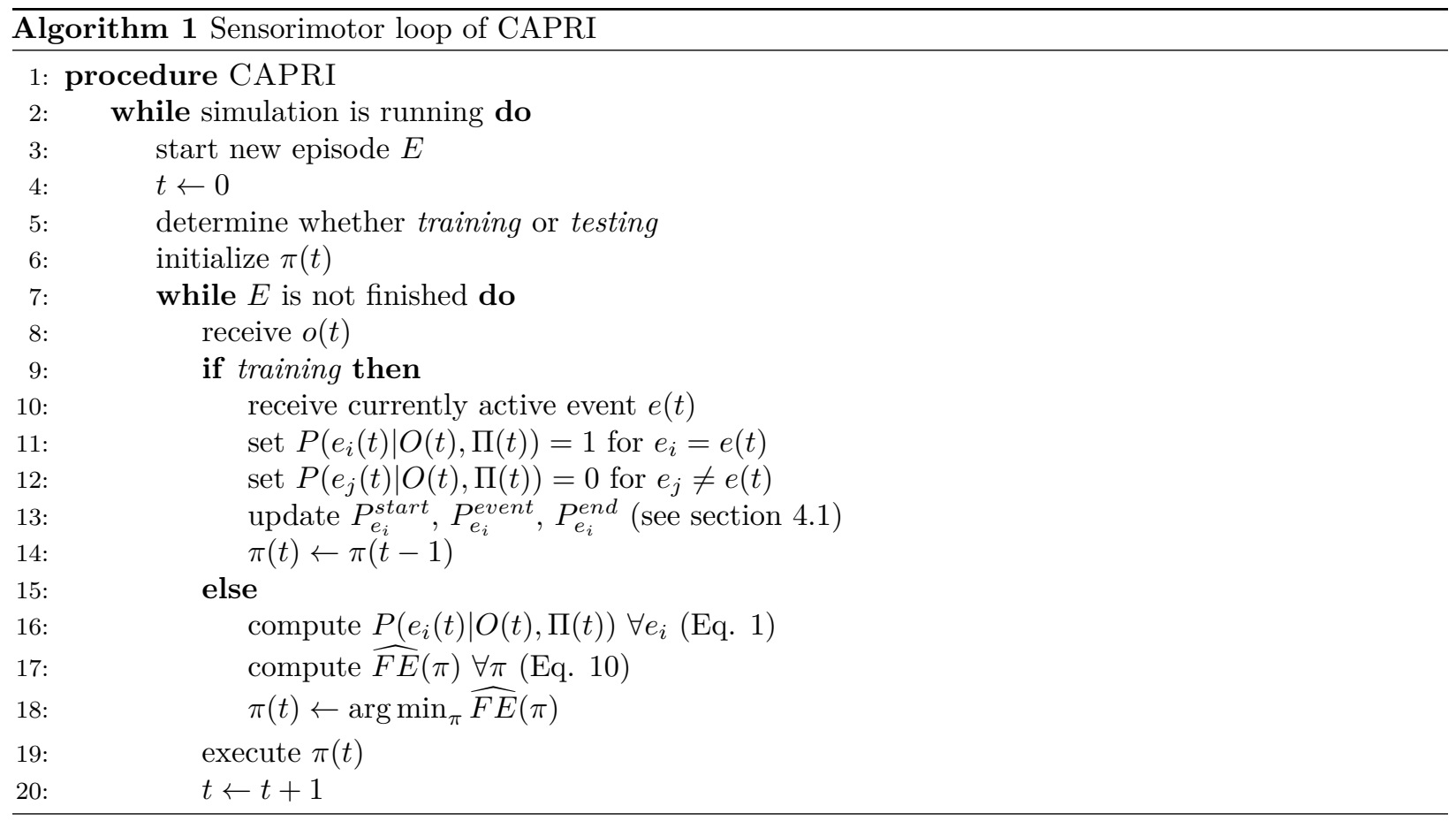

\subsection{Mixture Density Networks}

The goal of the subcomponents of each event schema is to model a distribution $P(y \mid x)$, with $y=o(t)$ the observation at time $t$ and $x$ some conditional variable. Because we assume that this distribution can locally be modeled as a multivariate Gaussian distribution, we want to allow that depending on $x$ the location and shape of the Gaussian distribution is allowed to change. This is why, in our current implementation, each likelihood distribution $P(y \mid x)$ is generated by a separate Mixture Density Network (Bishop, 2006). Each network receives $x$ as an input and produces $\bar{y}$ and $\Sigma_{y}$ as an output. The likelihood $P(y \mid x)$ can then be computed using $\mathcal{N}(y)(\bar{y}(x) \mid \Sigma(x))$.

The output neurons of $\bar{y}$ use a linear activation function. The output neurons for $\Sigma_{y}$ employ Exponential Linear Units (ELU) shifted by 1, to ensure that $\Sigma_{y}>0$. For simplicity we only use a diagonal matrix for $\Sigma_{y}$, which corresponds to the assumption that there is no covariance between the different dimensions of the observation. The network is trained using stochastic gradient descent with a learning rate of 0.001 . As a loss 
function $L$ we use negative log likelihood loss squashed by a tanh:

$$
L(x)=\tanh (-\log \mathcal{N}(y)(\bar{y}(x) \mid \Sigma(x) \cdot b) \cdot c .
$$

The squashing via the tanh-function avoids that a very low likelihood $(\approx 0)$ destroys the weights in the beginning of training. The empirically chosen constant factor $b$ scales the likelihood, such that little information is lost in the squashing process. The constant factor $c$ scales the loss before backpropagation and was set empirically. We use single-layered networks, seeing that a linear dependency between $x$ and $y$ is sufficient in our simulations. The weights were randomly initialized following a zero-centered Gaussian distribution with a standard deviation of 0.1 .

To speed up the training of the networks while avoiding local overfitting, we use a sampling buffer with a large capacity (1000 samples) for each network, as in Gumbsch et al. (2019). Every time the network is updated, two additional samples are drawn from the sampling buffer and the network is trained on these older samples. After updating the network with a new input $x$ and nominal outputs $y$, the input-output pair $(x, y)$ is added to the respective sampling buffer.

\subsection{Derivation of the active inference computation}

Our active inference computation is based on Friston et al. (2015). Since Friston et al. use different notations, we here outline how we derive Equation 6. Friston et al. compute the quality, or negative expected free energy, $\mathbf{Q}$ of a policy $\pi$ at time $t$ by

$$
\mathbf{Q}_{t}(\pi)=-E_{Q\left(s_{t} \mid \pi\right)}\left[H\left[P\left(o_{\tau} \mid s_{\tau}\right)\right]\right]-D_{\tau}\left[Q\left(o_{\tau} \mid \pi\right) \| P\left(o_{\tau} \mid m\right)\right]
$$

where $E$ denotes an expectation, $H$ the entropy, and $D$ the Kullback-Leibler divergence. $P$ and $Q$ denote probability and posterior distributions, respectively. Identical to our formulations, $o$ describes observations, $m$ a generative model of internal state preference, and $\tau$ a temporal horizon expanding into the future. However, Friston et al. use $s$ to describe internal states of the system, whereas we use $e$ instead to describe internal event estimations. Furthermore we do not mark time using a subscript. Thus we can reformulate the Equation in our notation as:

$$
\mathbf{Q}(\pi, t)=-E_{Q(e(t) \mid \pi)}[H[P(o(\tau) \mid e(\tau))]]-D_{\tau}[Q(o(\tau) \mid \pi) \| P(o(\tau) \mid m)] .
$$

Additionally we express the expectation $E$ over posterior distributions $Q(e(t) \mid \pi)$ as an expectation over events $e_{i}$ and over the temporal horizon $\tau$ :

$$
\mathbf{Q}(\pi, t)=-E_{e_{i}, \tau}\left[H\left[P\left(o(\tau) \mid e_{i}(\tau)\right)\right] Q\left(e_{i}(\tau) \mid \pi\right)\right]-D_{\tau}[Q(o(\tau) \mid \pi) \| P(o(\tau) \mid m)]
$$


Moreover, because the likelihood of an observation $o(\tau)$ does not only depend on the current event $e_{i}$, i.e. $P\left(o(\tau) \mid e_{i}(\tau)\right)$, but also on the current policy $\pi$, we make this dependency explicit, i.e. $P\left(o(\tau) \mid e_{i}(\tau), \pi\right)$. Thus, we get

$$
\mathbf{Q}(\pi, t)=-E_{e_{i}, \tau}\left[H\left[P\left(o(\tau) \mid e_{i}(\tau), \pi\right)\right] Q\left(e_{i}(\tau) \mid \pi\right)\right]-D_{\tau}[Q(o(\tau) \mid \pi)|| P(o(\tau) \mid m)] .
$$

Similarly, in our system the posterior of events does not only depend on the policy $\pi$, i.e. $Q\left(e_{i}(\tau) \mid \pi\right)$, but also on all previous observations $O(\tau)=(o(\tau), o(\tau-1), \ldots, o(0))$ and policies $\Pi(\tau)=(\pi(\tau), \pi(\tau-1), \ldots, \pi(0))$. This allows modeling complex scenarios with long-lasting events and dependencies between observations, actions, and future events. Hence, we replace $Q\left(e_{i}(\tau) \mid \pi\right)$ with $P\left(e_{i}(\tau) \mid O(\tau), \Pi(\tau)\right)$, which leads to

$$
\mathbf{Q}(\pi, t)=-E_{e_{i}, \tau}\left[H\left[P\left(o(\tau) \mid e_{i}(\tau), \pi\right)\right] P\left(e_{i}(\tau) \mid O(\tau), \Pi(\tau)\right)\right]-D_{\tau}[Q(o(\tau) \mid \pi)|| P(o(\tau) \mid m)] .
$$

Along similar lines we assume that the next observation $o(t+1)$ does not only depend on the current policy $\pi(t)$ but also on the current event $e_{i}(t)$. Thus, we replace $Q(o(\tau) \mid \pi)$ with $P\left(o(\tau) \mid e_{i}(\tau), \pi\right) P\left(e_{i}(\tau) \mid O(\tau), \Pi(\tau)\right)$, which is the posterior for an observation $o(t)$ given event $e_{i}$ and policy $\pi$ weighted by the estimated probability of event $e_{i}$. As a result we get

$$
\begin{aligned}
\mathbf{Q}(\pi, t)= & -E_{e_{i}, \tau}\left[H\left[P\left(o(\tau) \mid e_{i}(\tau), \pi\right)\right] P\left(e_{i}(\tau) \mid O(\tau), \Pi(\tau)\right)\right] \\
& -D_{e_{i}, \tau}\left[P\left(o(\tau) \mid e_{i}(\tau), \pi\right) P\left(e_{i}(\tau) \mid O(\tau), \Pi(\tau)\right) \| P(o(\tau) \mid m)\right] .
\end{aligned}
$$

Finally, if we consider minimizing positive expected free energy $\widehat{F E}$ as

$$
\widehat{F E}(\pi)=-\mathbf{Q}(\pi, t)
$$

we end up with Equation 6.

\subsection{Simulation details}

Here we provide further details on the simulation environment, complementary to Section 5.1. In our simulation the system received an 18-dimensional real-valued sensory observation with:

$$
o=\left[x_{x}^{a}, x_{y}^{a}, x_{z}^{a}, v_{x}^{a}, v_{y}^{a}, v_{z}^{a}, s^{a}, x_{x}^{p}, x_{y}^{p}, x_{z}^{p}, v_{x}^{p}, v_{y}^{p}, v_{z}^{p}, s^{p}, x_{x}^{p-a}, x_{y}^{p-a}, x_{z}^{p-a}, d^{p-a}\right],
$$

with $x$ positions, $v$ velocities, $s$ the shape, and $d$ the distance. Superscript letters denote to which entity the quantity refers to, with $a$ denoting agent, $p$ denoting patient, and $p-a$ denoting agent relative to patient. Subscript letters denote $x-, y-$, or $z$-dimension of positions and velocities. $x-$ and $y$-positions and velocities are scaled such that they are $\in[-1,1]$. $z$-position, shape, and distance are scaled to be $\in[0,1]$.

The policy $\pi$ was represented by a three-dimensional one hot encoding. During training for each new 
event sequence a policy was randomly sampled and the system executed this policy over the whole event sequence. During testing, the system always started with the no fixation policy $\pi_{n}$, which is active in the first time step, and then inferred its gaze policy starting with the second time step.

During training, the starting positions of agent and patient were randomly sampled before each new event sequence, ensuring that agent and patient were sufficiently distant $\left(d^{p-a}>0.1\right)$. Additionally, the patient started always on the ground, with $x_{z}^{p}=0$. For all events involving agent motions, the velocity was uniformly randomly sampled before each event with $v_{a} \in[0.01,0.05]$. For $e_{\text {reach }}$ and $e_{\text {transport }}$ this velocity remained constant during the event. For randomly directed motion, we simulated a simplified version of friction: the velocity decreased over time by a fixed factor of 0.97 .

To guarantee that the testing event sequences had the same duration and were comparable across simulations, the starting position and velocities were not sampled over the whole space for $E_{\text {test }}$. The patient was always positioned at the center of the screen $\left(x^{p}=[0,0,0]\right)$. The agent started above the floor and close to one corner of the screen $\left(x_{x}^{a} \in\{-0.6,0.6\}, x_{y}^{a} \in\{-0.6,0.6\}, x_{z}^{a}=0.6\right)$. The velocity for reaching was always set to $v^{a}=0.01$. The direction and velocity of $e_{\text {random }}$ was randomly determined.

The different event types had different criteria that caused the events to end: Standing still ended after 100 simulation time steps. Randomly directed motion ended when the velocity of the agent was approximately zero $\left(v^{a}<0.0005\right)$. Reaching ended when the distance between agent and patient was smaller than a threshold $\left(d^{p-a}<0.05\right)$. Transportation ended when a goal position, which was randomly generated before the start of the event, was reached, with the distance being smaller than a threshold (0.05).

The shape feature of hand or claw was randomly sampled for every simulation. The appearance of a hand could be $\in[0,0.5)$. The appearance of a claw could be $\in(0.5,1.0]$. The idea for this is that we do not want to make any assumptions on how differently hands and claws were perceived. In this way we randomize the difference of visual appearance across simulations, and appearance difference lies $\in(0,1]$. Note that setting the shape of a hand agent to $s^{a}=0$ and the shape of a claw agent to $s^{a}=1$ yielded very similar results (not shown) for both the inferred event probabilities as well as the gaze behavior. However, when setting the agents' appearance fixed, there was less variation across different simulations.

\subsection{Experiment 2}

In a second experiment we further investigated how experience with reaching affects the goal-anticipatory gaze of the CAPRI system. Thus, we manipulated how often the system encountered the event sequence $E_{\text {grasp }}$, which consisted of $e_{\text {grasp }}$ followed by $e_{\text {transport }}$ and $e_{\text {random }}$, during training. We tested five conditions that differ in the percentage of $E_{\text {grasp }}$ sequences in the training phase: The system was trained on $0.1 \%, 1 \%, 10 \%$, $20 \%$ and $50 \% E_{\text {grasp }}$ sequences of overall 1000 training event sequences. We ran this experiment 10 times per condition with different random initializations of the model weights and simulation parameters. After training, we tested the system on $10 E_{\text {test }}$ sequences with a hand- or claw agent for each simulation. 
Time $t$ of the first activation of $\pi_{p}$

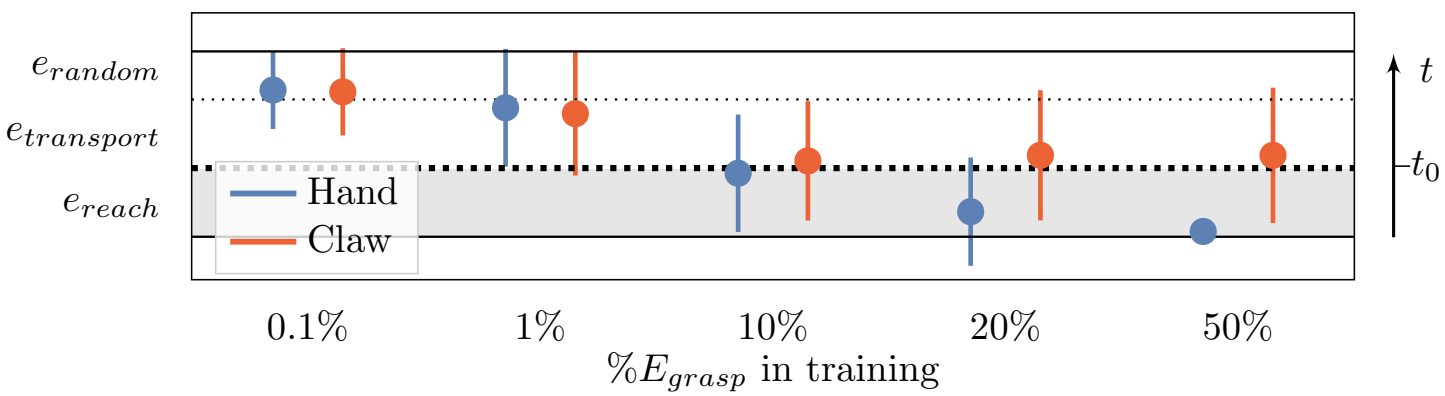

Figure 8: The gaze behavior for CAPRI during the test phases over the experience with reaching. We plot the mean time $t$ during an event sequence $E_{\text {test }}$ when the system first activated policy $\pi_{p}$, which corresponds to looking at the patient, over percentual training experience with grasping sequences $E_{\text {grasp }}$. The $x$-axis shows the percentage of experience with $E_{\text {grasp }}$ out of 200 training sequences. The $y$-axis shows time during one testing sequence $E_{\text {test }}$ from bottom to top with $t_{0}$ marking the arrival of the agent at the patient. Blue points show the gaze when the system observed a hand agent; red depict the results for a claw agent. Error bars show the standard deviation. Data points in the gray area mark anticipatory gaze behavior.

\subsubsection{Results of Experiment 2}

Figure 8 shows the point in time $t$ when CAPRI looked at the target, i.e., activated $\pi_{p}$, during the $E_{\text {test }}$ sequence for the first time in the different conditions. With more experiences of $E_{\text {grasp }}$ the system tended to look at the patient earlier. For $0.1 \%$ experience with $E_{\text {grasp }}$ the system on average activated $\pi_{p}$ at the beginning of the random motion event $e_{\text {random }}$. For $1 \%$ experience with $E_{\text {grasp }}$ the system started activating $\pi_{p}$ at the end of the transportation event $e_{\text {transport }}$ and for $10 \%$ experience at the beginning of $e_{\text {transport }}$. For $0.1-10 \%$ experience with grasping there was no clear difference for hand- or claw agents. For 10,20, and $50 \%$ experience with $E_{\text {grasp }}$, the first time of activating $\pi_{p}$ for claw agents hardly differs. For hand agents and $20 \%$ or more experience with $E_{\text {grasp }}$, the system activated $\pi_{p}$ shortly after the onset of the reaching event $e_{\text {reach }}$.

\subsubsection{Discussion of Experiment 2}

Experiment 2 showed that when CAPRI has little experience with reaching and transporting events $\left(e_{\text {reach }}\right.$, $\left.e_{\text {transport }}\right)$, the system does not show goal-anticipatory gaze. With increasing experience with those events, the system tends to look at the patient earlier, typically once it was able to identify the reaching event.

These results are in line with experimental findings on the goal-anticipation of young infants. 4-month-old infants, who have little to no experience in grasping themselves, do not show a goal-anticipatory gaze when watching reaching movements (Kanakogi \& Itakura, 2011). Instead they reactively track the moving hand until it has reached the target object. Similarly our system reactively tracked the hand during reaching if it has experienced less than $10 \%$ grasping events of a total of 1000 event sequences. The goal-anticipatory gaze becomes progressively more pronounced for hand agents with proportionally more experience in grasping. 


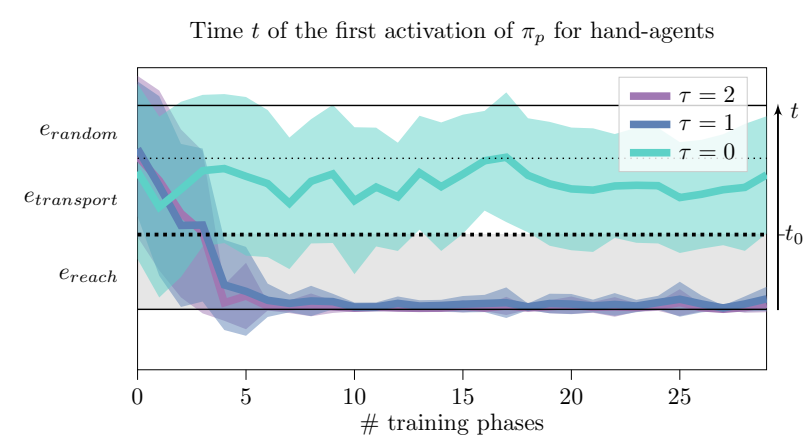

(a) Hand agents

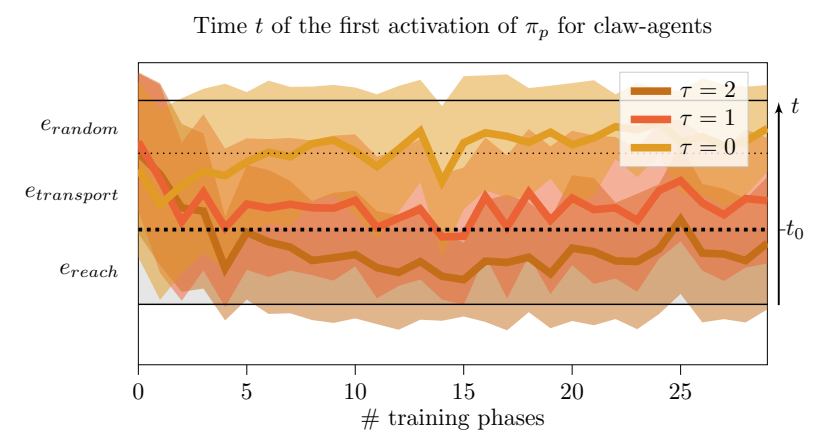

(b) Claw agents

Figure 9: Gaze behavior of CAPRI over experience and different values of $\tau$. We plot the mean time $t$ during an event sequence $E_{\text {test }}$ when the system first activated $\pi_{p}$, which corresponds to looking at the patient. The $x$-axis shows training experience in number of training phases. The $y$-axis shows time during one testing sequence $E_{\text {test }}$ from bottom to top with $t_{0}$ marking the arrival of the agent at the patient. (a) shows the gaze behavior for hand agents, (b) for claw agents. Colors illustrate different temporal horizons $\tau \in\{0,1,2\}$. Shaded areas show the standard deviation. Data points in the gray area mark anticipatory gaze behavior.

\subsection{Experiment 3}

In a third experiment, we investigated how the temporal horizon $\tau$ affects the gaze behavior of CAPRI. The horizon $\tau$ defines how many events into the future are considered when performing active inference to minimize predicted uncertainty. Here we tested values of $\tau \in\{0,1,2\}$. Again, the system was trained on 30 training phases with 100 event sequences per phase, each training phase was followed by a testing phase with $10 E_{\text {test }}$ sequences with hand- and claw agents, and we ran 20 simulations for each condition with different random initializations.

\subsubsection{Results of Experiment 3}

Figure 9 depicts when CAPRI first looked at the patient during the test phases, i.e., activated $\pi_{p}$ over training experience. Figure 9 (a) shows the gaze-behavior when hand agents perform $E_{\text {test }}$. If a temporal horizon of $\tau=0$ is chosen, the system first activated $\pi_{p}$ on average somewhere during $e_{\text {transport }}$ with large variance across simulations. Training experience does not visibly change the mean time of activating $\pi_{p}$ for $\tau=0$. Hence, for this parametric setting no goal-anticipatory gaze can be observed during reaching motions. For $\tau \geq 1$ the system systematically started activating $\pi_{p}$ right after the onset of $e_{\text {reach }}$ from the fourth training phase onwards when the movement is performed by hand agents.

Figure 9 (b) shows the gaze-behavior when claw agents perform $E_{\text {test }}$. With a temporal horizon of $\tau=0$ the system activated $\pi_{p}$ at the end of $e_{\text {transport }}$. For an increasing $\tau$, the system tended to first activate $\pi_{p}$ earlier during the sequence. For $\tau=1$ the system activated $\pi_{p}$ on average in the middle of $e_{\text {transport }}$ and for $\tau=2$ in the second half of $e_{\text {reach }}$. For claw agents and all settings of $\tau$ there was a large variance in gaze behavior across simulations. 


\subsubsection{Discussion of Experiment 3}

The results of Experiment 3 suggest that if the temporal horizon $\tau$ is sufficiently deep, an anticipatory gaze can be found for reaching events. For hand agents a temporal horizon of $\tau=1$ is sufficient, as already demonstrated by the previous experiment. For $\tau=1$ the system starts to consider not only the current event but also the next event boundary. This is why, once the system has identified $e_{\text {reach }}$, it looks at the patient because the position of the patient is highly informative for where the end of reaching will occur. This trend does not change for a deeper temporal horizon $(\tau=2)$.

When the reaching of a claw agent is observed, the system actually somewhat does perform goal-anticipatory gaze shifts for $\tau=2$. This stands in contrast to the fact that goal-anticipatory gaze shifts are typically not found for 11-month-old infants watching reaching motions of claw agents. Two interpretations are warranted. First, 11-month-old infants may have a restricted temporal horizon for anticipating the future. Indeed, previous research has shown that infants tend to only anticipate the first goal when observing a sequence of events with multiple goals: When 14-month-old infants observed movies of a hand first reaching for a target object and then placing this object in a container, anticipatory gazes towards the target object occurred during the reaching movement, but not towards the container during the subsequent transporting movement (Gredebäck, Stasiewicz, Falck-Ytter, Rosander, \& von Hofsten, 2009). This suggests that infants of that age tend to anticipate only one event boundary ahead in time. In our system this corresponds to a setting of $\tau=1$.

Second, out experimental results exhibit a rather large standard deviation. In many trials, the model does not generate anticipatory gaze shifts. The main explanation for the occurrence of anticipatory gaze shifts when $\tau=2$ and a claw is shown lies in the randomly sampled observations combined with the consideration of two possible event transitions. When a current observation fits rather well to a reaching motion, the system was likely to exhibit an anticipatory gaze shift because it then can expect a grasp, that is, the end condition of a reach at $\tau=2$. Due to the rather large number of computational iterations per event (100), such random transitions, although being unlikely, nonetheless tended to occur with high probability. Thus, goal-predictive gaze shifts seemed to occur randomly rather in a goal-directed manner.

While the results of Experiment 3 are largely in line with experimental findings, they also highlight the downsides of using a fixed temporal horizon for anticipating the future. In the case of high uncertainty about the current events, predictions of future event boundaries can become very unreliable. Thus, for future extensions of CAPRI, we want to consider a flexible, uncertainty-based temporal horizon. The goal is that the system can focus on predicting the immediate future in cases of high uncertainty. In the case of low uncertainty about the present the system could expand the temporal horizon and consider event boundaries farther distant in the future. 


\subsection{Experiment 4}

Experiment 1 demonstrated that CAPRI shows gaze behavior comparable to those of infants: When observing reaching events performed by agents with an 'hand-like' appearance $s^{a}=s_{\text {hand }}$, the system tends to look at the target of reaching, before reaching is concluded. For unfamiliar agents, also encoded by their shape $s^{a}$, the system tended to track the agent during the reaching motion. However, there were numerical differences between the experimental results when tracking the gaze of infants and our system. Infants tended to perform a goal-anticipatory gaze shift, for hand agents performing reaching motions, during the last $10 \%$ of the demonstrated event (Adam et al., 2016). In Experiment 1, our system performed a goal-anticipatory gaze right after the onset of the reaching event.

We hypothesize that these numerical differences can be attributed to some simplifications of our scenario. One explanation could be that our system experiences $E_{\text {test }}$ sequences exactly like the $E_{\text {grasp }}$ sequences that had occurred during training, while infants observe reaching movements from hands other than their own and on a screen. Thus, it may be harder for the infant to recognize the hand, and, as a result, take more time to recognize the reaching event and to perform the anticipatory gaze. To test this assumption, we systematically alter the perceived appearance of the hand agents for our system in Experiment 4.

For that we took the system of Experiment 1, which was trained on 30 training phases, and conducted additional testing phases, each composed of 10 testing sequences $E_{\text {test }}$ with hand agents. For each event sequence $E_{t e s t}$, we altered the perceived shape of the hand agent $\left(s^{a}\right)$ by an offset $\Delta s_{\sigma}^{a}$. The offset $\Delta s_{\sigma}^{a}$ was randomly sampled from a zero-centered Gaussian distribution with standard deviation $\sigma$ for each event sequence. We tested five different conditions with $\sigma \in[0.05,0.1,0.2,0.3,0.4,0.5]$. We analyzed how systematically altering the perception of the hand affects the policy inference.

\subsubsection{Results of Experiment 4}

Figure 10 shows the point in time $t$ when CAPRI first looked at the target, i.e., activated $\pi_{p}$, during the $E_{\text {test }}$ sequence with a hand agent. The $x$-axis shows different standard deviations $\sigma$ of the Gaussian distributions from which the shape modification $\Delta s_{\sigma}^{a}$ was sampled. For $\sigma \leq 0.1$ the system tends to activate $\pi_{p}$ right after the onset of $e_{\text {reach }}$. With increasing values of $\sigma$ the system tends to activate $\pi_{p}$ later during the reaching event. For $\sigma=0.5$ the system on average activated $\pi_{p}$ shortly before $e_{\text {reach }}$ is concluded. Besides delaying the goal-anticipatory gaze, larger shape modifications results in larger variations across simulations.

\subsubsection{Discussion of Experiment 4}

We hypothesized that the earlier occurrence of the anticipatory gaze of CAPRI in Experiment 1 compared to those of infants in eye-tracking experiments can partially be attributed to the similarity of training and testing for our system. Experiment 4 showed that, the goal-anticipatory gaze of our system occurred later during the reaching event of the $E_{\text {test }}$ event sequence when the perceived appearance of the hand was randomly altered during testing. The delay of the goal-anticipatory gaze increased with stronger modifications of the hand's 
Time $t$ of the first activation of $\pi_{p}$

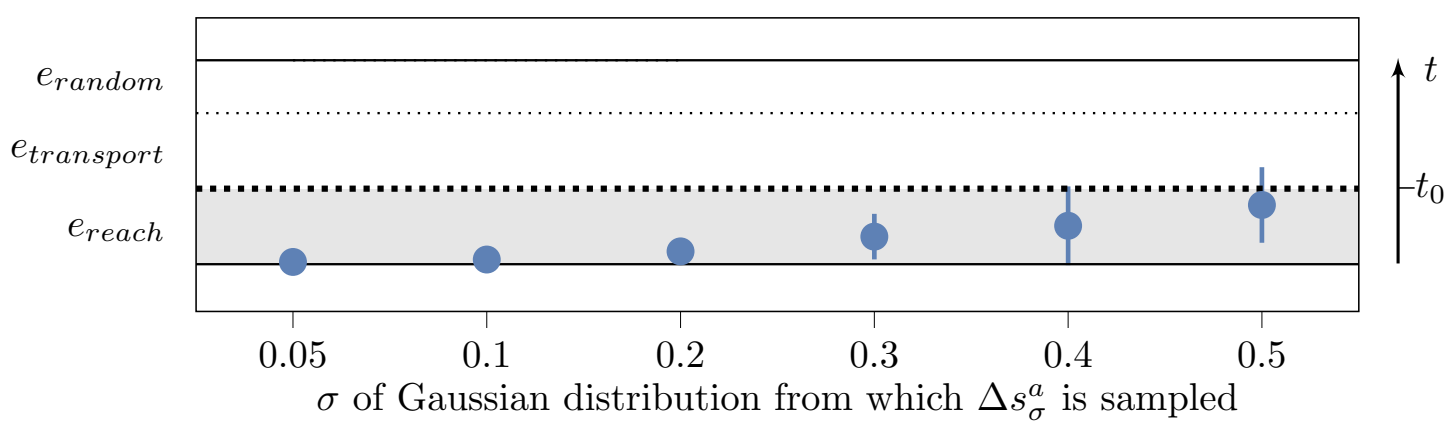

Figure 10: The gaze behavior for CAPRI when, after 30 training phases, the appearance of the hand is systematically modified. In each testing phase $s^{a}$ is modified by adding $\Delta s_{\sigma}^{a}$, which is sampled from a Gaussian distribution with standard deviation $\sigma$. We plot the mean time $t$ during an event sequence $E_{\text {test }}$ when the system first activated policy $\pi_{p}$, which corresponds to looking at the patient, over different standard deviations $\sigma$. The $y$-axis shows time during one testing sequence $E_{\text {test }}$ from bottom to top with $t_{0}$ marking the arrival of the agent at the patient. Error bars show the standard deviation.

appearance. This can be understood when focusing on the event inference of our system: For an agent with a shape that looks less 'hand-like' the system requires on average more observations to correctly infer that reaching is being observed. A later recognition of a reaching event results in a later goal-anticipatory gaze. 


\subsection{Derivation of event probability estimation}

Here we provide a full derivation of Equation 2:

$$
\begin{aligned}
& P\left(e_{i}(t) \mid o(t), o(t-1), \pi(t-1), e_{j}(t-1)\right)=\frac{P\left(e_{i}(t), o(t), o(t-1) \pi(t-1), e_{j}(t-1)\right)}{P\left(o(t), o(t-1), \pi(t-1), e_{j}(t-1)\right)} \quad \text { Bayes rule } \\
& =\frac{P\left(o(t) \mid o(t-1), \pi(t-1), e_{i}(t), e_{j}(t-1)\right) \cdot P\left(o(t-1), \pi(t-1), e_{i}(t), e_{j}(t-1)\right)}{P\left(o(t), o(t-1), \pi(t-1), e_{j}(t-1)\right)} \quad \text { Bayes rule } \\
& =\frac{P\left(o(t) \mid o(t-1), \pi(t-1), e_{i}(t), e_{j}(t-1)\right) \cdot P\left(o(t-1), \pi(t-1) \mid e_{i}(t), e_{j}(t-1)\right) \cdot P\left(e_{i}(t) \mid e_{j}(t-1)\right) \cdot P\left(e_{j}(t-1)\right)}{P\left(o(t), o(t-1), \pi(t-1), e_{j}(t-1)\right)}
\end{aligned}
$$

factoring the joint likelihood $P\left(o(t-1), \pi(t-1), e_{i}(t), e_{j}(t-1)\right)$ in numerator

$$
=\frac{P\left(o(t) \mid o(t-1), \pi(t-1), e_{i}(t), e_{j}(t-1)\right) \cdot P\left(o(t-1), \pi(t-1) \mid e_{i}(t), e_{j}(t-1)\right) \cdot P\left(e_{i}(t) \mid e_{j}(t-1)\right) \cdot P\left(e_{j}(t-1)\right)}{\sum_{e_{h}} P\left(o(t), o(t-1), \pi(t-1), e_{j}(t-1), e_{h}(t)\right)}
$$

marginalization of $P\left(o(t), o(t-1), \pi(t-1), e_{j}(t-1)\right)$ over $e_{h}$ in denominator

$$
=\frac{P\left(o(t) \mid o(t-1), \pi(t-1), e_{i}(t), e_{j}(t-1)\right) \cdot P\left(o(t-1), \pi(t-1) \mid e_{i}(t), e_{j}(t-1)\right) \cdot P\left(e_{i}(t) \mid e_{j}(t-1)\right) \cdot P\left(e_{j}(t-1)\right)}{\sum_{e_{h}} P\left(o(t) \mid o(t-1), \pi(t-1), e_{h}(t), e_{j}(t-1)\right) \cdot P\left(o(t-1), \pi(t-1) \mid e_{h}(t), e_{j}(t-1)\right) \cdot P\left(e_{h}(t) \mid e_{j}(t-1)\right) \cdot P\left(e_{j}(t-1)\right)}
$$

factoring the joint probability $P\left(o(t), o(t-1), \pi(t-1), e_{h}(t), e_{j}(t-1)\right)$ in denominator

$$
=\frac{P\left(o(t) \mid o(t-1), \pi(t-1), e_{i}(t), e_{j}(t-1)\right) \cdot P\left(o(t-1), \pi(t-1) \mid e_{i}(t), e_{j}(t-1)\right) \cdot P\left(e_{i}(t) \mid e_{j}(t-1)\right)}{\sum_{e_{h}} P\left(o(t) \mid o(t-1), \pi(t-1), e_{h}(t), e_{j}(t-1)\right) \cdot P\left(o(t-1), \pi(t-1) \mid e_{h}(t), e_{j}(t-1)\right) \cdot P\left(e_{h}(t) \mid e_{j}(t-1)\right)}
$$

cancelling $P\left(e_{j}(t-1)\right)$ in numerator and denominator

$$
=\frac{P\left(o(t) \mid o(t-1), \pi(t-1), e_{i}(t), e_{j}(t-1)\right) \cdot P\left(o(t-1), \pi(t-1) \mid e_{j}(t-1)\right) \cdot P\left(e_{i}(t) \mid e_{j}(t-1)\right)}{\sum_{e_{h}} P\left(o(t) \mid o(t-1), \pi(t-1), e_{h}(t), e_{j}(t-1)\right) \cdot P\left(o(t-1), \pi(t-1) \mid e_{j}(t-1)\right) \cdot P\left(e_{h}(t) \mid e_{j}(t-1)\right)}
$$

Markov Assumption: $P\left(o(t-1), \pi(t-1) \mid e_{h}(t), e_{j}(t-1)\right)=P\left(o(t-1), \pi(t-1) \mid e_{j}(t-1)\right)$

$$
=\frac{P\left(o(t) \mid o(t-1), \pi(t-1), e_{i}(t), e_{j}(t-1)\right) \cdot P\left(e_{i}(t) \mid e_{j}(t-1)\right)}{\sum_{e_{h}} P\left(o(t) \mid o(t-1), \pi(t-1), e_{h}(t), e_{j}(t-1)\right) \cdot P\left(e_{h}(t) \mid e_{j}(t-1)\right)}
$$

cancelling $P\left(o(t-1), \pi(t-1) \mid e_{j}(t-1)\right)$ in numerator and denominator 